\title{
Electrodynamics in a very thin current sheet leading to magnetic reconnection
}

\author{
N. Singh ${ }^{1,2}$, C. Deverapalli ${ }^{2}$, and G. Khazanov ${ }^{3}$ \\ ${ }^{1}$ ECE, University of Alabama in Huntsville, Huntsville, AL 35899, USA \\ ${ }^{2}$ CSPAR, University of Alabama in Huntsville, Huntsville, AL 35899, USA \\ ${ }^{3}$ NSSTC/MSFC, Huntsville, AL35812, USA
}

Received: 20 December 2005 - Revised: 12 June 2006 - Accepted: 21 June 2006 - Published: 22 September 2006

\begin{abstract}
We study the formation of a very thin current sheet (CS) and associated plasma electrodynamics using threedimensional (3-D) particle-in-cell simulations with ion to electron mass ratio $\mathrm{M} / \mathrm{m}=1836$. The $\mathrm{CS}$ is driven by imposed anti-parallel magnetic fields. The noteworthy features of the temporal evolution of the CS are the following: (i) Steepening of the magnetic field profile $\mathrm{B}_{\mathrm{x}}(\mathrm{z})$ in the central part of the CS, (ii) Generation of three-peak current distribution with the largest peak in the CS center as $\mathrm{B}_{\mathrm{x}}(\mathrm{z})$ steepens, (iii) Generation of converging electric fields forming a potential well in the CS center in which ions are accelerated. (iv) Electron and ion heating in the central part of the CS by currentdriven instabilities (CDI). (v) Re-broadening of the CS due to increased kinetic plasma pressure in the CS center. (vi) Generation of electron temperature anisotropy with temperature perpendicular to the magnetic field being larger than the parallel one. (vii) Current disruption by electron trapping in an explosively growing electrostatic instability (EGEI) and electron tearing instability (ETI). (viii)The onset of EGEI coincides with an increase in the electron temperature above the temperature of the initially hot ions as well as the appearance of new shear in the electron drift velocity. (ix) Bifurcation of the central CS by the current disruption. (x) Magnetic reconnection (MR) beginning near the null in $\mathrm{B}_{\mathrm{x}}$ and spreading outward. (xi) Generation of highly energized electrons reaching relativistic speeds and having isotropic pitch-angle distribution in the region of reconnected magnetic fields. We compare some of these features of the current sheet with results from laboratory and space experiments.
\end{abstract}

\section{Introduction}

Formation of thin current sheets (TCS) of widths of the order of ion skin depth $\left(\lambda_{\mathrm{i}}\right)$ is now confirmed by satellite observations (Pulkkinnen et al., 1993, 1994; Sergeev et al., 2003,

Correspondence to: N. Singh

(singh@ece.uah.edu)
1998; Hoshino et al., 1996; Runov et al., 2003a, b). The stability of such current sheets has been extensively studied (e.g., see Büchner and Zelenyi, 1987; Pellat et al., 1991; Lakhina et al., 1993). It is becoming increasingly evident that the TCS might have current profiles significantly different from that in a Harris current sheet (CS) (Harris, 1962). Bifurcated current sheets (BCS) have been observed from Geotail (Hoshino et al., 1996) and more recently from Cluster (Baumjohan and Nakamura, 2002; Runov et al., 2003a, b). The cluster satellites have also revealed CSs with triple peaks having a sharp peak in the center and two side peaks; rectangular and asymmetric shapes have been also observed (Nakamura et al., 2004; Zelenyi, 2004). In view of such observations new theoretical studies on the formation of thin current sheets are appearing (Sitnov et al., 2003; Zelenyi et al., 2004). These studies construct one-dimensional solutions of relevant plasma equations for a steady-state CS including the anisotropy of electron and/or ion temperatures.

Besides TCSs having thickness of the order of $\lambda_{i}$, now even thinner current sheets have been reported from satellite observations having thickness of the order of the electron skin depth. Wygant et al. (2005) recently reported observations from Cluster that extremely thin current sheets (ETCS) of widths as thin as few times the electron skin depth $\left(\lambda_{\mathrm{e}}\right)$ are embedded within the TCSs of scale size $\lambda_{\mathrm{i}}$. The TCSs perhaps are embedded in even wider current sheets. Wygant et al. (2005) reported the following features of ETCSs in the magneto-tail: (i) the widths of ETCSs are a few electron skin depth $\left(\sim 3 \lambda_{\mathrm{e}}\right)$ and they are embedded in a wider CS of thickness $\left(\sim 40 \lambda_{\mathrm{e}}\right)$; (ii) the ETCSs are bifurcated with a current disruption in the middle; (iii) the embedded ETCSs are colocated with a deep negative potential well and (iv) the unmagnetized ions in the CS are accelerated into the potential well. We have previously reported such features of ETCSs from 3-D kinetic simulations (Singh, 2004) including its formation and subsequent bifurcation due to current disruption by current-driven instabilities.

Published by Copernicus GmbH on behalf of the European Geosciences Union and the American Geophysical Union. 
There are now numerous simulation studies of magnetic reconnection and current sheets. These simulations extend in variety from MHD to hybrid and fully kinetic particle-in-cell (PIC) simulations. Both 2-D and 3-D simulations are available now. In order to induce reconnection in MHD simulations anomalous resistivity is included using heuristic models (Ugai et al., 1986). In 2-D PIC (2-DPIC) simulations the so-called the third dimension, along which the current flows, the plasma dynamics are ignored. In this category there is a rich body of numerous studies, which have greatly contributed to the physics of magnetic reconnection (Hesse et al., 1998, 1999, 2002; Shay et al., 1998, 2003; Rogers et al., 2001). Major results of such 2-DPIC simulations are the realization that electron pressure anisotropy (Hesse et al., 1998; 1999), whistler waves and kinetic Alfvén waves play key role in mediating reconnection in thin current sheets with scale size less than $\sim \lambda_{\mathrm{i}}$ (Rogers et al., 2001; Shay et al., 1998). Another set of 2-D simulations have shown that, in an initialized current sheet lower hybrid drift instability (LHDI) first occurs in the outer regions where the density gradients are large. The LHDI triggers drift kinetic instability (DKI) in the central part of the CS affecting magnetic reconnection (Ozaki et al., 1996; Silin and Büchner, 2003a, b; Horiuchi and Sato, 1999). 3DPIC simulations of current sheets are reported by Horiuchi and Sato (1999), Zeiler et al. (2002), Drake et al. (2003), Singh (2004) and Pritchett and Coroniti (2004). Horiuchi and Sato compared the reconnection with and without a convection electric field showing that when the convection electric field penetrates into the neutral sheet region reconnection might occur, otherwise the LHDI followed by DKI triggers reconnection. In the work of Zeiler et al. (2002), Drake et al. (2003) and Pritchett and Coroniti (2004) the nonlinear evolution of the magnetic reconnection was studied in response to initialized magnetic field perturbations reconnecting the anti-parallel magnetic field lines. These simulations with guide fields revealed interesting nonlinear features of plasma and fields as the initialized reconnection progressed; some of the noteworthy features are the generation of parallel electric fields, parallel acceleration of electrons, electron hole turbulence and density cavity formation. Cattell et al. (2005) have shown that in 2-D PIC simulations electron holes also form if the size of the simulation box is sufficiently long along the anti-parallel magnetic fields. Scholer et al. (2003) reported 3-D simulations with and without a guide field demonstrating that in a CS of scale size $\lambda_{\mathrm{i}}$ reconnection processes are driven by lower hybrid drift instabilities (LHDI) without the help of DKI; for this purpose they used a sufficiently high ion to electron mass ratio $\mathrm{M} / \mathrm{m}=150$. The nonlinear processes associated with LHDI were further studied by Doughton et al. (2004) in a 2-D simulation with a mass ratio $M / m=1836$, showing that LHDI introduces substructures in the current distribution due to the electron dynamics in the CS. The substructure includes bifurcation of the CS by enhancement of the current near the current sheet edges.
Singh (2004) studied the formation of a ETCS of scale size $\left(\sim \lambda_{\mathrm{e}}\right)$ in response to an externally applied anti-parallel magnetic field to an initially current-free uniform plasma. He found that current-driven electrostatic instabilities occur in an ETCS and affect the reconnection explosively, when the current disrupts due to the trapping of the current-carrying electrons. The trapping produces large anomalous resistivity accompanying the reconnection. The localized current disruption in the central CS bifurcates the ETCS in two distinct current sheets. In retrospect we find that such features of the current sheet seen in the simulation (Singh, 2004) have features of ETCSs reported from Cluster (Wygant et al., 2005); the observed bifurcated CS of scale size $\sim \lambda_{\mathrm{e}}$ have nearly zero current at the center and not nearly the initial maximum current as determined without the effect of LHDI in the 2-D simulation of Doughton et al. (2004). The Cluster observation of bifurcated ETCSs with zero current at the center having similarity with our previous simulation has prompted us to do similar simulations with certain improvements as discussed below.

In our previous simulation we used an artificially low ion to electron mass ratio $\mathrm{M} / \mathrm{m}=16$. We point out that most of the recent 3-D simulations of reconnection use artificially low mass ratio. For example, Horiuchi and Sato (1999), Zeiler et al. (2002), Drake at al. (2003) and Pritchett and Coroniti (2004) used $M / m=100$. This lowering of the ion mass significantly reduces the ion cyclotron period and introduces the ion-cyclotron dynamics in the simulation at a much faster time scale than might occur in real situations. This might shorten the time when only the electron-cyclotron dynamics might dominate in the formation and evolution of current sheets. Thus, electron-MHD (EMHD) type of flows consisting of magnetized electrons and un-magnetized ions in current sheets could be relevant for a longer time period even on spatial scales comparable to the ion Larmor radius after the introduction of the magnetic perturbations, which initiate magnetic reconnection. Consequences of such limitations of artificially low ion to electron mass ratio remain largely unexplored. We have performed a new set of simulations with $\mathrm{M} / \mathrm{m}=1836$ and also with different scale sizes of the external anti-parallel magnetic field, which drives the simulations. The purpose of this paper is to present results from these new simulations focusing on the following points: (i) Thinning of a CS forming ETCS, (ii) Formation of multiple peaks in the current distribution, (iii) Generation of electric fields normal to the CS plane, (iii) Ion acceleration by the normal electric fields, (iv) The nature of the current-driven electrostatic instabilities inside the CS, (v) Onset of electron tearing mode and magnetic reconnection (MR) near the magnetic field null, (vi) Outward expansion of the MR-associated electromagnetic plasma turbulence, and (vii) Electron and ion acceleration including production of relativistic isotropic electron population in the CS. We point out that the first three items mentioned here are essentially the features of EMHD flows. We compare the features of the current sheets seen in 

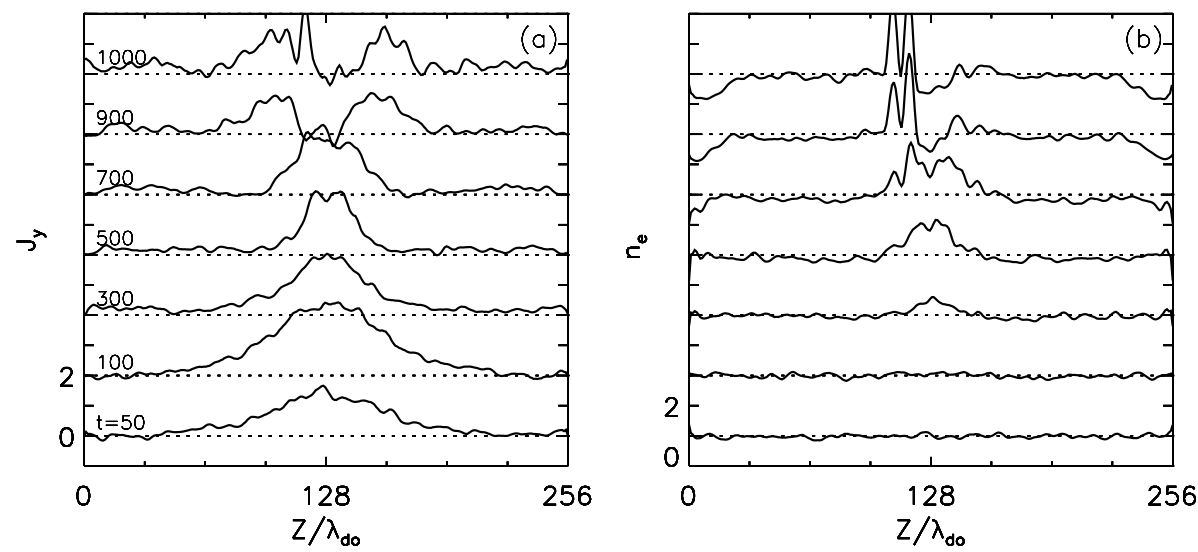

Fig. 1. Temporal evolution of the distribution of (a) current $\mathrm{J}_{\mathrm{y}}(\mathrm{z})$ and (b) electron density $\mathrm{n}_{\mathrm{e}}$. Times are labeled in units of $\omega_{\text {po }}^{-1}$. The dotted horizontal lines are separated by $2 \mathrm{~J}_{\text {th }}$ in the current panel and $2 \mathrm{n}_{\mathrm{O}}$ in the density panel. Initial normalized density is unity.

the simulations with those observed from Cluster (Wygant et al., 2005).

\section{Simulation method}

Our simulation method is described in detail in Singh (2004). We simulate a 3-D volume of plasma of size $\mathrm{L}_{\mathrm{x}} \times \mathrm{L}_{\mathrm{y}} \times \mathrm{L}_{\mathrm{z}}$. We impose anti-parallel magnetic field

$\mathbf{B}_{\mathrm{xo}}=B_{0} \tanh \left[\left(z-L_{z} / 2\right) / L\right] \mathbf{a}_{x}$.

Initially at time $t=0$, the simulation box is filled with a uniform plasma with Maxwellian electrons and ions having ion and electron temperatures $T_{i}=2 T_{e}$ and $T_{e}=T_{0}$, respectively. This initial condition allows us to study the processes involved in the formation of a current sheet. In many theoretical and simulation studies, it is common to use an initial equilibrium with pressure balance across the CS. This initial equilibrium is either unstable due to some inherent instability processes due to the structure of the current sheet and electron and ion drifts in it or it is made unstable by artificially introducing perturbations. The initial conditions used in this paper presents a continuum of processes occurring both during and after the $\mathrm{CS}$ formation including magnetic reconnection. In this paper we have limited to extremely thin current sheets of scale size $L$ of the order of a few electron skin depth $\lambda_{\mathrm{e}}$.

In previously reported simulations using a 3-D electromagnetic particle-in-cell (3-D EMPIC) code, we had chosen $\mathrm{L}=\lambda_{\mathrm{e}}$ and studied the instabilities in such a thin current sheet (Singh, 2004) with ion to electron mass $M / m=16$. In the simulations presented here we have varied $L$ from $\lambda_{\mathrm{e}}$ to $4 \lambda_{\mathrm{e}}$ in a proton plasma having ion to electron mass ratio $\mathrm{M} / \mathrm{m}=1836$. The simulation with the largest value of $\mathrm{L}$ in (Eq. 1) revealed interesting plasma processes affecting the dynamical evolution of a thin CS from one form of current distribution to another. Since recent Cluster observations have begun to reveal
CS with scale size of few times $\lambda_{\mathrm{e}}$ (Nakamura et al., 2004; Wygant et al., 2005) in the Earth's magnetotail, we believe the physics revealed by our simulations is relevant to space plasma physics dealing with the complex MR problem. We used $\mathrm{L}_{\mathrm{x}}=\mathrm{L}_{\mathrm{y}}=32 \lambda_{\mathrm{do}}$ and $\mathrm{L}_{z}=256 \lambda_{\mathrm{do}}$, where $\lambda_{\text {do }}$ is the Debye length in the initial plasma. The magnetic field $\mathrm{B}_{\mathrm{o}}$ in Eq. (1) is chosen to yield maximum electron cyclotron frequency $\Omega_{\mathrm{eo}}=0.5 \omega_{\mathrm{po}}$, where $\omega_{\mathrm{po}}$ is the electron plasma frequency with the initial density $n_{0}$. The numerical grid size in all three dimensions is $\lambda_{\text {do }}\left(\Delta x=\Delta y=\Delta \mathrm{z}=\lambda_{\text {do }}\right)$ and each cubic cell of this size contains 25 pairs of electrons and ions as macroparticles. We treat the evolution of the plasma and fields by solving the equations of motions of the macro-particles and Maxwell equations using particle-in-cell method (PIC) (Birdsall and Langdon, 1985).

For future reference, we point out the following spatial and temporal scales in the simulation: electron skin depth $\lambda_{\mathrm{e}}=10 \lambda_{\mathrm{do}}$, ion skin depth $\lambda_{\mathrm{i}}=428 \lambda_{\mathrm{do}}$, electron Larmor radius $\rho_{e} \geq 2 \lambda_{\mathrm{do}}$, ion Larmor radius $\rho_{\mathrm{i}} \geq 120 \lambda_{\mathrm{do}}$, electron plasma pe$\operatorname{riod} \tau_{p e}=6.28 \omega_{\mathrm{po}}^{-1}$, electron cyclotron period $\tau_{c e}=12.5 \omega_{\mathrm{po}}^{-1}$, ion plasma period $\tau_{p i}=270 \omega_{\mathrm{po}}^{-1}$ and ion cyclotron period $\tau_{c i}=22950 \omega_{\mathrm{po}}^{-1}$. Simulation is advanced in time with a time step $\Delta \mathrm{t}=0.02 \omega_{\mathrm{po}}^{-1}$. The ion-acoustic and Alfvén speeds are $\mathrm{C}_{s}=0.06 \mathrm{~V}_{\text {teo }}$ and $\mathrm{V}_{\mathrm{A}}=0.12 \mathrm{~V}_{\text {teo }}$, where $\mathrm{V}_{\text {teo }}$ is the electron thermal velocity with electron temperature $T_{0}$ in the initial plasma.

\section{Numerical results}

We performed three simulations with (i) $\mathrm{L}=12 \lambda_{\mathrm{do}}=1.2 \lambda_{\mathrm{e}}$, (ii) $\mathrm{L}=24 \lambda_{\mathrm{do}}=2.4 \lambda_{\mathrm{e}}$ and (iii) $\mathrm{L}=36 \lambda_{\mathrm{do}}=3.6 \lambda_{\mathrm{e}}$ and all have $\mathrm{M} / \mathrm{m}=1836$. The simulation with $\mathrm{L}=12 \lambda_{\mathrm{do}}$ yielded results quite similar to that reported previously in Singh (2004), except that evolution was slowed down by the large ion mass. We mainly discuss here the results from the third run with $\mathrm{L}=36 \lambda_{\mathrm{do}}=3.6 \lambda_{\mathrm{e}}$, which reveals the thinning of the current 


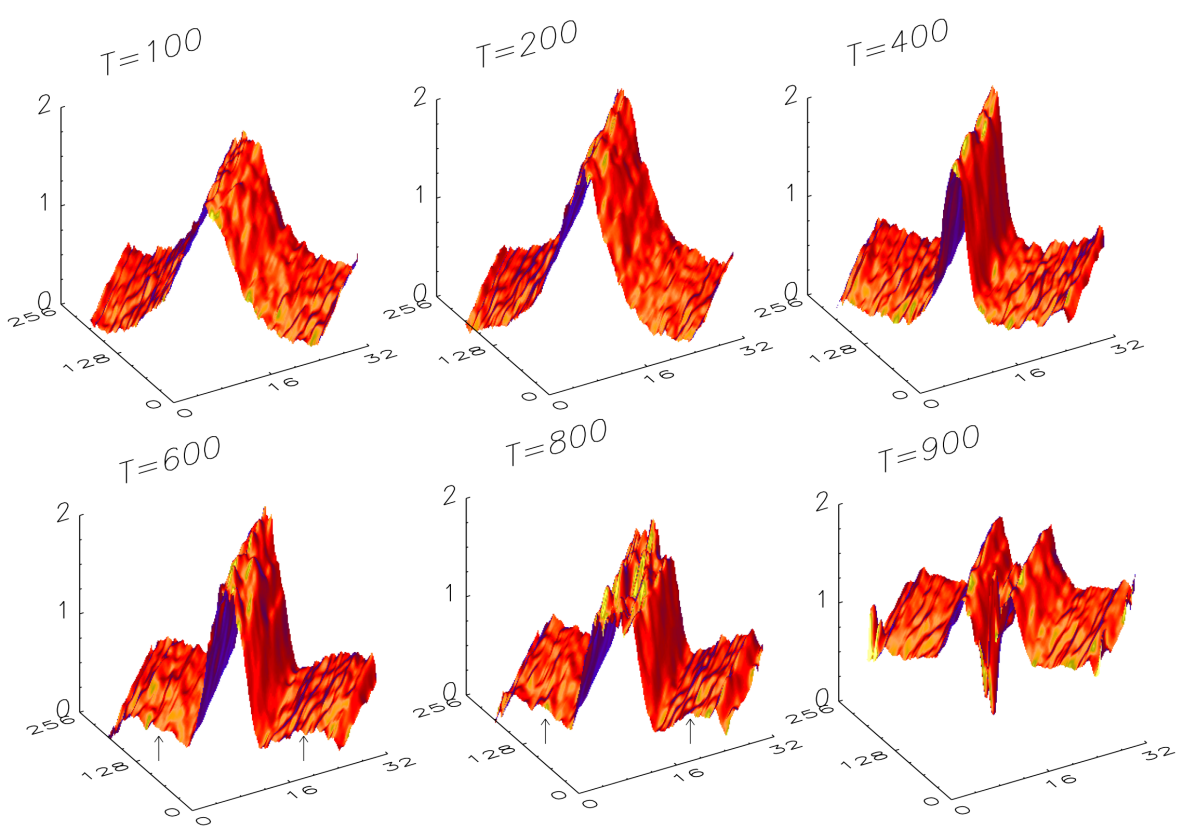

Fig. 2. Evolution of current distribution $J_{y}(z)$ is shown by surface plots at some selected times as labeled. Note that $J_{y}(z)$ first grows, then its distribution thins, develops secondary peaks as indicated by arrows. At even later times it re-broadens and the central peak bifurcates.

sheet and associated processes more clearly than the other two runs with already thin current sheets.

\subsection{Current sheet formation and thinning}

We first summarize the salient features of the evolution of the current distribution in a current sheet in response to the magnetic field being imposed on the plasma at $t=0$. This is followed by a detailed discussion on the plasma processes involved in the evolution and their effects on the plasma in the CS. Figure 1a shows the evolution of the current distribution over a time period up to $\mathrm{t}=900 \omega_{\mathrm{po}}^{-1}$. The corresponding evolution of the density profile is shown in Fig. 1b. Figure 1a shows $\mathrm{J}_{\mathrm{y}}(\mathrm{z})$ averaged over the $\mathrm{x}-\mathrm{y}$ plane. As early as $\mathrm{t}=50 \omega_{\mathrm{po}}^{-1}$ a current sheet has developed with peak current density $\mathrm{J}_{\mathrm{ym}} \sim 1.5 \mathrm{~J}_{\mathrm{th}}$, where $\mathrm{J}_{\mathrm{th}}=\mathrm{n}_{\mathrm{o}} \mathrm{eV}_{\text {teo }}$, the thermal current density in the plasma with initial density $\mathrm{n}_{\mathrm{o}}$ and electron thermal velocity $V_{\text {teo }}$. Since initially we have $\mathrm{J}_{\mathrm{y}}=0$, it is natural to ask how does this current arise? In the simulation, the main cause for the initiation of the current is the generation of an electric field $\mathrm{E}_{\mathrm{y}}$, given by the Maxwell equation

$\partial \mathrm{E}_{\mathrm{y}} / \partial \mathrm{t}=\left(\mu_{\mathrm{o}} \varepsilon_{\mathrm{o}}\right)^{-1}\left(\partial \mathrm{B}_{\mathrm{x}} / \partial \mathrm{z}\right)-\varepsilon_{\mathrm{o}}^{-1} \mathbf{J}_{\mathrm{y}}$.

When $\mathrm{J}_{\mathrm{y}} \sim 0$, the magnetic field gradient generates $\mathrm{E}_{\mathrm{y}}$, which accelerates electrons especially in the region of low $B$ and large electron cyclotron periods. As soon as the electrons begin to accelerate in -y direction, they feel $\mathbf{J} \times \mathbf{B}$ force causing the generation of Hall electric field $\mathrm{E}_{\mathrm{z}}$ pointing toward the CS center as explained later in detail. Then the $\mathbf{E}_{z} \times \mathbf{B}_{x}$ drift maintains the current. The electric field $\mathrm{E}_{\mathrm{y}}$ initiating the current is seen to last until $\mathrm{t} \sim 200 \omega_{\mathrm{po}}^{-1}$. This transient $\mathrm{E}_{\mathrm{y}}$ plays the same role in the simulation as the induced electric fields in the experiments of Syrovatskii et al. $(1973,1981)$ and Stenzel and Gekelman (1981).

By the time $\mathrm{t}=100 \omega_{\mathrm{po}}^{-1}, \mathrm{~J}_{\mathrm{ym}}$ has grown to $\sim 2.2 \mathrm{~J}_{\mathrm{th}}$ (Fig. 1a) and the current distribution has a single peak. The plots at $\mathrm{t}=300 \omega_{\mathrm{po}}^{-1}$ and $500 \omega_{\mathrm{po}}^{-1}$ show that the main peak in the current distribution has considerably thinned. The thinning of the CS is more clearly seen in Fig. 2, which is a surface plot of $\mathrm{J}_{\mathrm{y}}(\mathrm{x}$, $\mathrm{y}=16, \mathrm{z})$ in the $\mathrm{x}-\mathrm{z}$ plane. We see that the current sheet is the thinnest at $\mathrm{t} \sim 400 \omega_{\text {po }}^{-1}$ and it is beginning to develop additional weaker peaks on both sides of the large-amplitude main peak. The additional secondary peaks, as indicated by the arrows, are well developed at $\mathrm{t}=600$ and $800 \omega_{\mathrm{po}}^{-1}$ with amplitudes $\sim 20 \%$ of the main central peak. The three-peak feature of the composite CS continues until the main central peak bifurcates after $t \geq 900 \omega_{\mathrm{po}}^{-1}$. Note that before the bifurcation of the main peak, it widens for $t \geq 600 \omega_{\mathrm{po}}^{-1}$. Also note the growth of the large-amplitude spatial oscillations in $\mathrm{J}_{\mathrm{y}}(\mathrm{z})$ for $\mathrm{t}>400 \omega_{\mathrm{po}}^{-1}$.

Figure $1 \mathrm{~b}$ shows the evolution of electron density $\mathrm{n}_{\mathrm{e}}(\mathrm{z})$. The curves in this figure correspond to $\mathrm{J}_{\mathrm{y}}(\mathrm{z})$ in Fig. 1a. Ion density, $\mathrm{n}_{\mathrm{i}}(\mathrm{z})$, follows $\mathrm{n}_{\mathrm{e}}(\mathrm{z})$ except when large-amplitude oscillations set in the fields and the current $\mathrm{J}_{\mathrm{y}}(\mathrm{z})$ at late times as seen in Fig. 2. Note the increase in the plasma density by nearly a factor of two in the central region while the density has decreased in the outer region after $\mathrm{t} \sim 400 \omega_{\mathrm{po}}^{-1}$. This readjustment of the density distribution implies transport of plasma from the outer to the inner regions of the CS; the mechanism of the transport is discussed later. 

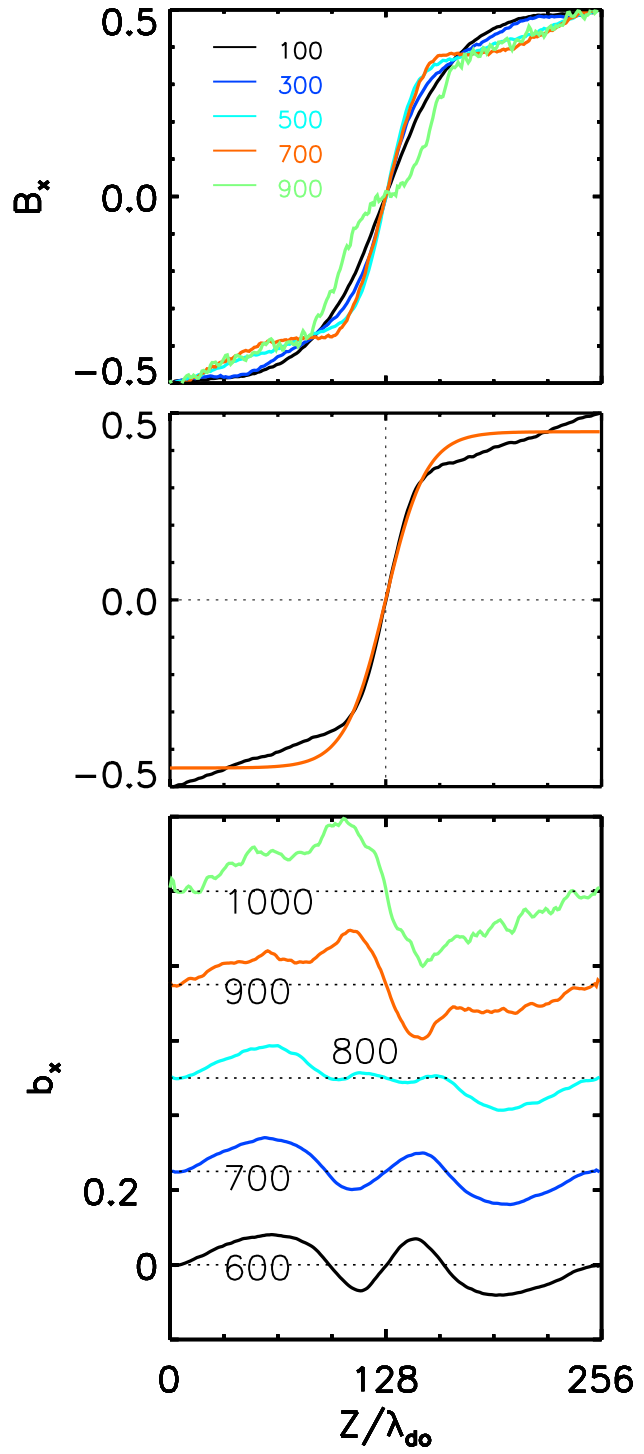

Fig. 3. (a) Evolution of $B_{x}(z)$ corresponding to the evolution of $\mathrm{J}_{\mathrm{y}}(\mathrm{z})$ in Fig. 1. Note the steepening of $\mathrm{B}_{\mathrm{x}}(\mathrm{z})$ in the CS central region. (b) The steepened $\mathrm{B}_{\mathrm{X}}(\mathrm{z})$ at $\mathrm{t}=500 \omega_{\text {po }}^{-1}$ and a fit of Harris distribution shown by the red line. (c) The magnetic field perturbation $\mathrm{b}_{\mathrm{x}}(\mathrm{z})$ affecting $\mathrm{B}_{\mathrm{x}}(\mathrm{z})$ in $(\mathrm{a})$.

Figure 3a shows the evolution of the CS as manifested in the magnetic field distribution $\mathrm{B}_{\mathrm{x}}(\mathrm{z})$, which is plotted at selected times. The curve (black) at $\mathrm{t}=100 \omega_{\mathrm{po}}^{-1}$ is nearly the same as the initial profile given in (1). The subsequent profiles from $\mathrm{t}=300 \omega_{\mathrm{po}}^{-1}$ to $700 \omega_{\mathrm{po}}^{-1}$ (blue to red) show that $\mathrm{B}_{\mathrm{X}}(\mathrm{z})$ is progressively steepened in the central region over $80<\mathrm{z} / \lambda_{\mathrm{do}}<176$, which is about $\delta \mathrm{z} \sim 9.6 \lambda_{\mathrm{e}}$ wide, where $\lambda_{\mathrm{e}}$ is the electron skin depth $\left(\lambda_{\mathrm{e}} \sim 10 \lambda_{\mathrm{do}}\right)$. The steepening is achieved by the increase in $B_{x}$ in the immediate neighborhood of the central line $\mathrm{z}=128$ and decreases further away near the boundaries $\mathrm{z}=0$ and $256 \lambda_{\mathrm{do}}$. The increased slope of $\mathrm{B}_{\mathrm{x}}(\mathrm{z})$ in the central part and the decreased slopes in the

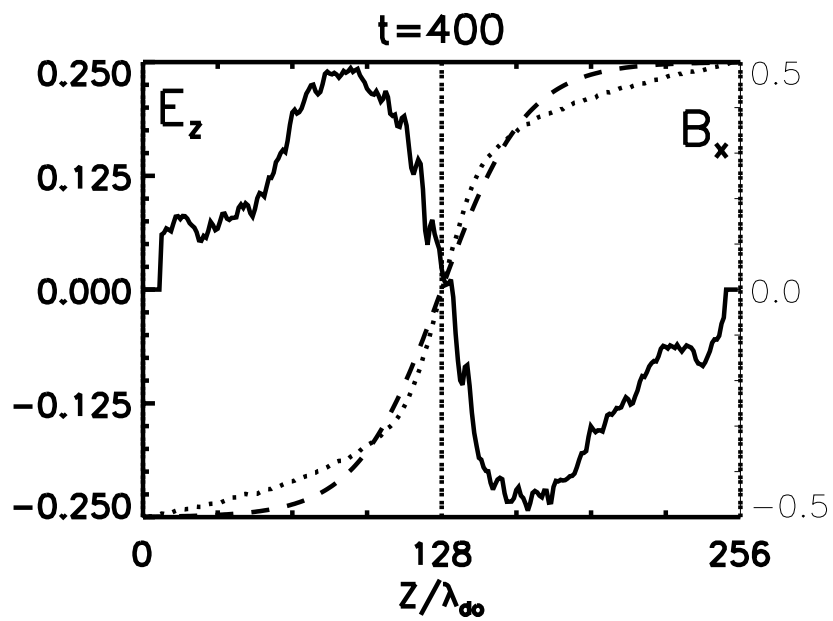

Fig. 4. Electric field $E_{Z}(z)$ (solid line), steepened $B_{X}(z)$ (dotted line) and initial $\mathrm{B}_{\mathrm{X}}(\mathrm{z})$ (long-dashed line).

outer regions represents the three-peak current sheet in Fig. 2 for $600 \omega_{\mathrm{po}}^{-1}<\mathrm{t}<800 \omega_{\mathrm{po}}^{-1}$. We have fitted a Harris distribution given by $\mathrm{B}=0.4 \tanh \left[\left(\mathrm{z}-\mathrm{L}_{z} / 2\right) / \mathrm{L}_{t}\right)$ to the steepened central part of the profile as shown in Fig. $3 \mathrm{~b}$; we find that scale size $L_{t} \sim 24 \lambda_{\text {do }}$ gives a nice fit in contrast to $L=36 \lambda_{\text {do }}$ in the imposed field profile in Eq. (1). The steepened profile in the center yields a maximum current density $\mathrm{J}_{\mathrm{ymax}}=$ $\mu_{\mathrm{o}}^{-1} \nabla \times\left.\mathbf{B}\right|_{\max }=2 \mathrm{~J}_{\text {th }}$ as seen in Fig. 1a, in contrast to the maximum current $\mathrm{J}=1.39 \mathrm{~J}_{\text {th }}$ with $\mathrm{L}=36 \lambda_{\text {do }}$ in Eq. (1).

The steepened profile $B_{x}(z)$ in Fig. $3 b$ is the sum of the imposed field $B_{x o}(z)$ in Eq. (1) and magnetic field perturbations $b_{x}$, that is, $B_{x}(z)=B_{x o}(z)+b_{x}(z)$. Temporal evolution of $b_{x}(z)$ is shown in Fig. 3c. Note that until $t \sim 700 \omega_{p o}^{-1}$ the signs of the perturbations are such that, the total field decreases in the outer regions but very close to the center, on either side of it, the field is enhanced. It appears that magnetic flux is transported from outer regions to the region near the center. At $t=800 \omega_{\mathrm{po}}^{-1}$ the inner perturbations in $\mathrm{b}_{\mathrm{x}}(\mathrm{z})$ near the center vanish and the current sheet is then re-widening. At later times shown in Fig. 3c, the perturbation $b_{x}$ has become bipolar and it cancels the imposed field yielding $\mathrm{B}_{\mathrm{x}}(\mathrm{z}) \sim 0$ and $\mathrm{J}_{\mathrm{y}}(\mathrm{z}) \sim 0$ over an extended part of the central CS, as apparent from the bifurcation of the current seen at $\mathrm{t}=900 \omega_{\mathrm{po}}^{-1}$ in Figs. 1a and 2.

Why does the thinning occur? We find that the $\mathbf{J}_{\mathrm{y}} \times \mathbf{B}$ force on the magnetized electrons drives the thinning. This force facilitates the transport of plasma toward the CS center, as mentioned earlier in connection with the evolution of the plasma density in Fig. 1b. The possibility of this plasma transport is easily seen from the momentum equations for the electrons and ions. If we ignore the electron inertia in the electron momentum equation it gives the z-component of the electric field

$E_{\mathrm{z}}=\left(1 / \mathrm{en}_{\mathrm{e}}\right) \mathbf{J}_{\mathrm{y}} \times \mathbf{B}_{\mathrm{x}}-\left(1 / \mathrm{en}_{e}\right) \partial\left(\mathrm{n}_{\mathrm{e}} \mathrm{T}_{\mathrm{ez}}\right) / \partial \mathrm{z}$. 

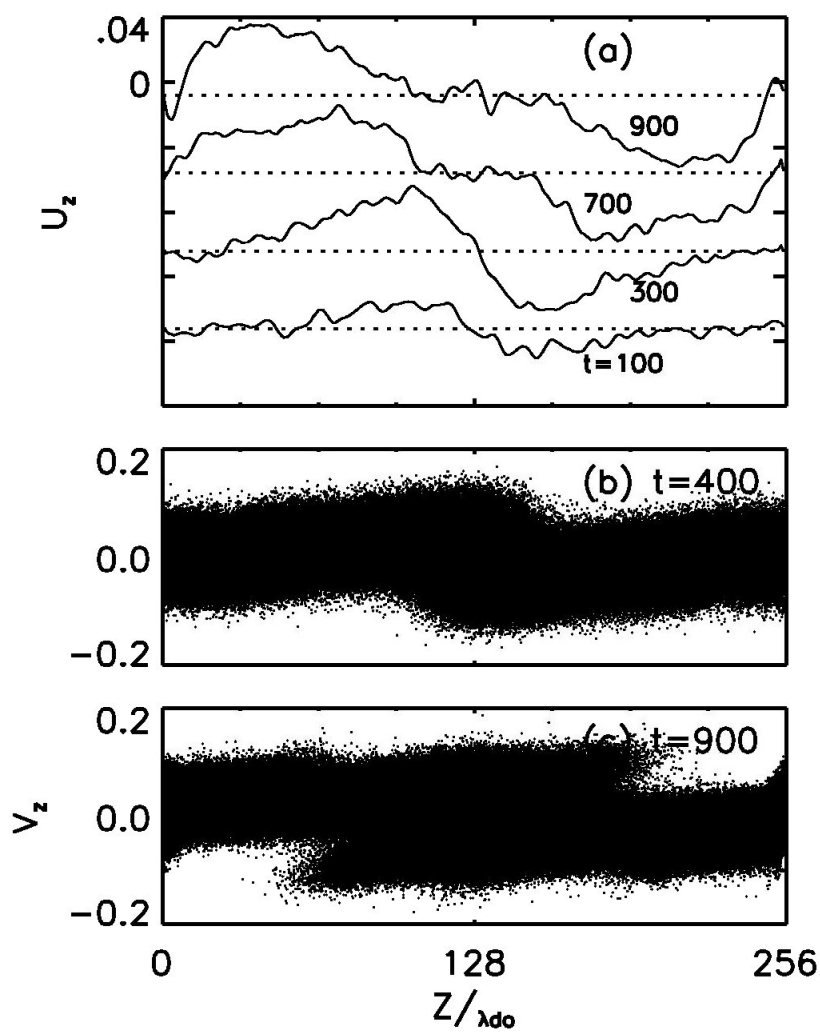

Fig. 5. Ions' inflow: (a) Evolution of the average flow velocity U(z) is shown from $t=200 \omega_{\text {po }}^{-1}$ to $t=900 \omega_{\text {po }}^{-1}$ as labeled. Ion phase space in $\mathrm{z}-\mathrm{V}_{z}$ plane at (b) $\mathrm{t}=400 \omega_{\text {po }}^{-1}$ and $(\mathbf{c}) \mathrm{t}=900 \omega_{\text {po }}^{-1}$.

In the initial stages the pressure term in Eq. (3) is insignificant and the $\mathbf{J}_{\mathrm{y}} \times \mathbf{B}$ force determines $\mathrm{E}_{\mathrm{z}}$. For the magnetic field in Eq. (1) $E_{z}$ represents converging electric fields pointing toward the CS center. Figure 4 shows $\mathrm{E}_{\mathrm{z}}(\mathrm{z})$ at $\mathrm{t}=400 \omega_{\mathrm{po}}^{-1}$ along with $\mathrm{B}_{\mathrm{x}}(\mathrm{z}) . \mathrm{E}_{\mathrm{z}}$ accelerates the un-magnetized ions toward the CS center; this acceleration is given by

$M\left(\partial \mathrm{U}_{\mathrm{z}} / \partial \mathrm{t}+\mathrm{U}_{\mathrm{z}} \partial \mathrm{U}_{\mathrm{z}} / \partial \mathrm{z}\right) \approx \mathrm{eE}_{\mathrm{z}}-\left(1 / \mathrm{n}_{\mathrm{i}}\right) \partial\left(\mathrm{n}_{\mathrm{i}} \mathrm{T}_{\mathrm{iz}}\right) / \partial \mathrm{z}$.

In Eqs. (3) and (4) $\mathrm{T}_{\mathrm{iz}}$ and $\mathrm{T}_{\mathrm{ez}}$ are the ion and electron temperatures associated with their motions in the $\mathrm{z}$ direction, respectively. The evolution of the average ion flow velocity $U_{Z}$ is shown in Fig. $5 a ; U_{z}$ reaches a maximum value $\mathrm{U}_{\max } \sim 0.04 \mathrm{~V}_{\text {teo }}=1.7 \mathrm{~V}_{\text {tio }}$, where $\mathrm{V}_{\text {tio }}$ is the initial ion thermal velocity. The transport of the plasma towards the center increases the plasma density as shown in Fig. 1b. Another consequence of the ion acceleration by $E_{z}$ is that the ions counter-stream in the central region of the CS. The counterstreaming begins during the early stage of the thinning and continues even after the reconnection sets-in the CS. Figures $5 \mathrm{~b}$ and $\mathrm{c}$ show ion phase space in the $\mathrm{z}-\mathrm{V}_{\mathrm{z}}$ plane; at an early time $\mathrm{t}=400 \omega_{\mathrm{po}}^{-1}$ the flows of the accelerated ions originating from top and bottom parts of the CS have just begun to overlap while at a late time $\mathrm{t}=900 \omega_{\mathrm{po}}^{-1}$ the flows are overlapping over an extended central region.
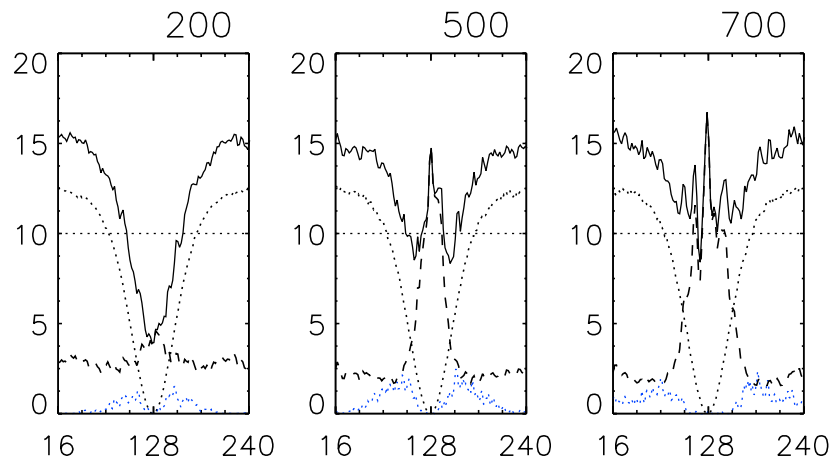

Fig. 6. Evolution of pressure distribution: from left to right the panels show magnetic $\mathrm{P}_{\mathrm{m}}(\mathrm{z})$ (dotted line), kinetic $\mathrm{P}_{\mathrm{k}}(\mathrm{z})$ (dashed line) and total pressure $\mathrm{P}_{\mathrm{t}}(\mathrm{z})$ (solid line) at $\mathrm{t}=200 \omega_{\text {po }}^{-1}, \mathrm{t}=500 \omega_{\text {po }}^{-1}$ and $\mathrm{t}=700 \omega_{\text {po }}^{-1}$, respectively. $\mathrm{P}_{\mathrm{k}}(\mathrm{z})$ is the sum of electron and ion pressures.

Since the ions accelerated into the central region by $\mathrm{E}_{\mathrm{z}}$ merge with the preexisting ions there, the average velocity $U_{z}$ in Fig. 5a is much lower than that expected from the falling of the ions in the potential well associated with $\mathrm{E}_{\mathrm{z}}$. Integrating $\mathrm{E}_{\mathrm{z}}$ in Eq. (3) by ignoring the pressure term, the maximum potential drop associated with $\mathrm{E}_{\mathrm{z}}$ is

$\varphi_{a x} \sim\left(1 / \mathrm{en}_{\mathrm{o}}\right)\left(\mathrm{B}_{\mathrm{o}}{ }^{2} / 2 \mu_{\mathrm{o}}\right)=12.5 \mathrm{~T}_{\mathrm{o}} / \mathrm{e}$

From the simulation we find that $\varphi_{\max } \sim 17 \mathrm{~T}_{\mathrm{o}} / \mathrm{e}$, which is larger than the above theoretical value. The difference is attributed to space charges developing in the simulation, which yield $\mathrm{E}_{\mathrm{z}}$ larger than that expected from Eq. (3), especially near the boundaries. Maximum velocity of ions falling into a potential well of magnitude $\varphi_{\max } \sim 17 \mathrm{~T}_{\mathrm{o}} / \mathrm{e}$ is $\mathrm{V}_{\text {imax }}=0.136 \mathrm{~V}_{\text {teo }}$, which is also seen from Fig. $5 \mathrm{~b}$. Wygant et al. (2005) reported acceleration of ions scaling with the potential drop in Eq. (5) from Cluster observations. However, it is important to note that Wygant et al. noted the entire ion populations accelerated to the energy level in Eq. (5); this could be possible when the neutral region of the CS is deeply depleted of plasma before the CS formed. We find that $\mathrm{V}_{\text {imax }}$ is approximately the same as the magnetosonic wave propagation velocity $\mathrm{V}_{\mathrm{F}}=\left(\mathrm{C}_{\mathrm{S}}^{2}+\mathrm{V}_{\mathrm{A}}^{2}\right)^{1 / 2}$ and the thinning time $\tau_{\text {thin }}$ is about the transit time of ions across the half of the total width of the CS, namely,

$\tau_{\text {thin }} \sim 5 \lambda_{\mathrm{e} / \mathrm{V}_{\mathrm{F}}} \sim 50 \lambda_{\mathrm{do}} / 0.13 \mathrm{~V}_{\text {teo }} \sim 400 \omega_{\mathrm{po}}^{-1}$.

\subsection{Pressure balance and stoppage of the thinning}

One of the consequences of the formation of the thin current sheet is the energization of both electrons and ions. We just discussed that the ions are energized in the $\mathrm{z}$ direction by the bipolar electric field $E_{\mathrm{z}}$ (Fig. 4). Furthermore, currentdriven instabilities (CDIs) also heat both the electrons and ions, but with the electron heating being dominant as it occurs starting at an early phase of the CS evolution. We will 
discuss the features of the instabilities in the next section. Here we highlight that a consequence of electron and ion heating and the enhanced plasma density (Fig. 1b) is that the kinetic plasma pressure, $\mathrm{P}_{\mathrm{k}}$, progressively increases in the central CS. When $P_{k}$ becomes sufficiently large, the thinning stops and the central CS begins to re-broaden. Figure 6 shows the evolution of the pressures across the CS. As labeled on top, the panels from left to right give the pressures at an early time, $\mathrm{t}=200 \omega_{\mathrm{po}}^{-1}$, at the time when the central CS is almost the thinnest at $\mathrm{t}=500 \omega_{\mathrm{po}}^{-1}$, and at a later time when the thinned CS has begun to re-broaden, $\mathrm{t}=700 \omega_{\mathrm{po}}^{-1}$, respectively. In these panels the dotted, dashed and solid-line curves give the magnetic pressure $P_{m}(z)=B_{x}^{2} / 2 \mu_{0}$, the total kinetic pressure $\mathrm{P}_{\mathrm{k}}=\mathrm{P}_{\mathrm{ki}}+\mathrm{P}_{\mathrm{ke}}$ with $\mathrm{P}_{\mathrm{ki}}=\mathrm{n}_{\mathrm{i}} \mathrm{T}_{\mathrm{iz}}$ and $\mathrm{P}_{\mathrm{ke}}=\mathrm{n}_{\mathrm{e}} \mathrm{T}_{\mathrm{ez}}$, and the total pressure $\mathrm{P}_{t}=\mathrm{P}_{\mathrm{m}}+\mathrm{P}_{\mathrm{k}}$, respectively. Note that $\mathrm{T}_{\mathrm{iz}}$ and $\mathrm{T}_{\mathrm{ez}}$ are the ion and electron temperatures associated with their motion in the $\mathrm{z}$ direction. The curves in blue near the bottoms of the panels in Fig. 6 show the dynamic pressure associated with the average flow velocity $\mathrm{U}_{\mathrm{i}}(\mathrm{z})$ as shown in Fig. 5a. The dynamic pressure remains small due to the averaging including the background ions as explained earlier. $\mathrm{P}_{\mathrm{m}}(\mathrm{z})$ has a maximum value of $\mathrm{P}_{\mathrm{m}}=12.5 \mathrm{n}_{\mathrm{o}} \mathrm{T}_{\mathrm{o}}$ at the boundaries $z=0$ and $256 \lambda_{\text {do }}$ as determined by $B_{o}$ in Eq. (1), and it is zero at the center $\mathrm{z}=128 \lambda_{\mathrm{do}}$. We notice that $\mathrm{P}_{\mathrm{m}}(\mathrm{z})$ progressively decreases in the outer regions near the boundaries while it steepens on either side of the central region developing a shock-like ledge, in conformity of the evolution of $\mathrm{B}_{\mathrm{x}}(\mathrm{z})$ in Fig. 3a. $\mathrm{P}_{k}$ is seen to increase dramatically in the central region peaking near $\mathrm{z} \sim 128 \lambda_{\mathrm{do}}$. Note that in calculating $\mathrm{P}_{\mathrm{k}}$, effective temperatures of electrons and ions are used and they are given by the second moment of their velocity distribution functions. We find that by the time $t=500 \omega_{\mathrm{po}}^{-1}$ the peak kinetic pressure near the center becomes nearly equal to the total pressure $\mathrm{P}_{\mathrm{t}}=\mathrm{P}_{\mathrm{m}}+\mathrm{P}_{\mathrm{k}}$ near the edges of the simulation box. However, by this time the plasma instabilities in the central CS begin to produce fine structures in the $\mathrm{P}_{\mathrm{k}}$ profile.

The second moment of the electron and ion velocity distributions inside the current sheet yield the following effective temperatures at $\mathrm{t}=500 \omega_{\mathrm{po}}^{-1}: \mathrm{T}_{\mathrm{ez} / \mathrm{To}}=2.5 \mathrm{~T}_{\mathrm{o}}$ and $\mathrm{T}_{\mathrm{iz}}=4.8 \mathrm{~T}_{\mathrm{o}}$. With such temperatures and the enhanced density $\mathrm{n}(\mathrm{z}=128) \cong 2$ (Fig. $1 \mathrm{~b})$, the kinetic pressure at the CS center is $P_{k}=(5+9.6) n_{0} T_{0}=14.6$. The magnetic pressure $\mathrm{P}_{\mathrm{m}}(\mathrm{z}=0)=\mathrm{P}_{\mathrm{m}}(\mathrm{z}=256)=\mathrm{B}_{\mathrm{o}}^{2} / 2 \mu_{\mathrm{o}} \sim 12.5 \mathrm{n}_{\mathrm{o}} \mathrm{T}_{\mathrm{o}}$ and the kinetic pressure $P_{k}$ at $z=0$ and 256 is $\sim 2.5 n_{0} T_{0}$, giving total pressure at the boundaries $P_{t}=15$, which is nearly the same as $P_{k}$ at the center. When this pressure balance occurs the CS thinning stops.

We point out that the enhanced ion pressure, $P_{k i}=9.6 n_{0} T_{0}$, contributes significantly more than the electron pressure, $P_{k e}=5 n_{0} T_{0}$, to the pressure balance near the center at the time of the stoppage of the thinning. As mentioned earlier, the ion pressure enhancement is due to the ion acceleration by the normal electric field $E_{\mathrm{Z}}$ (Fig. 4) and the merging of the ion streams as shown in Fig. 5. However, as the CDI
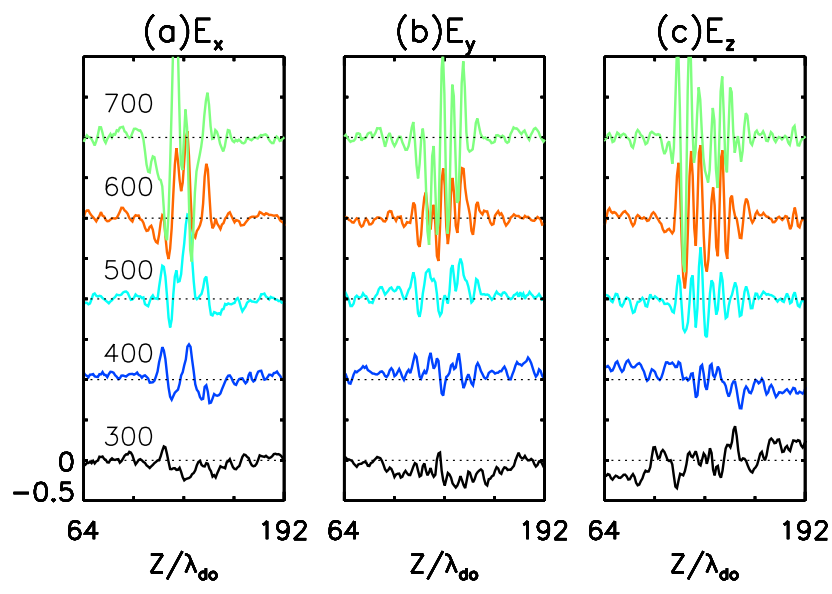

Fig. 7. Evolution of the fluctuations in (a) $E_{X}(z)$, (b) $E_{y}(z)$ and (c) $E_{z}(z)$ from $t=300 \omega_{\text {po }}^{-1}$ to $t=\omega_{\text {po }}^{-1}$ as labeled. Note that the fluctuations are confined in the central region of the current sheet.

continues to heat the electrons, the pressure $\mathrm{P}_{\mathrm{ke}}$ continues to increase and then the central peak in the CS broadens before it bifurcates due to current disruption.

\subsection{Electrostatic plasma turbulence}

We now examine the temporal and spatial behavior of the plasma instability, which drastically affects the CS and also leads to fast reconnection. Figures $7 \mathrm{a}-\mathrm{c}$ show the temporal evolution of electric fields $E_{x}(z), E_{y}(z)$ and $E_{z}(z)$ over the time interval from $t=300 \omega_{\text {po }}^{-1}$ to $t \sim 700 \omega_{\text {po }}^{-1}$. We find that the instability in the current sheet starts out as purely electrostatic (ES) and remains so until $\mathfrak{t} \sim 850 \omega_{\text {po }}^{-1}$ when magnetic reconnection and electron tearing instability begin to occur. Comparing the phases of the growing fluctuations in the electric field components at the successive times shown in Fig. 7, we find that the ES instability is nearly purely growing; spatially static (non-propagating) structures in the fields and the plasma density grow in time.

The spatial structure of the instability is shown in Figs. 8a$c$, in which $E_{x}, E_{y}$, and $E_{z}$ are plotted in the $x-z(y=16)$ (top) and $\mathrm{x}-\mathrm{y}(\mathrm{z}=128)$ (bottom) planes, respectively. As already noted from Fig. 7, the growing ES oscillations are confined in the central region of the CS over $112<\mathrm{z} / \lambda_{\mathrm{do}}<144$ as seen from the top panels in Fig. 8. The wavelength along $\mathrm{z}$ is $\lambda_{z} \sim 8 \lambda_{\text {do }}$, giving a wave number $\mathrm{k}_{z}=2 \pi / \lambda_{z}=0.7$. The wave structure in the $\mathrm{x}-\mathrm{y}$ plane is more complicated; it spears to be superposition of different modes.

The evolution of the electrostatic instability in the wave number plane $\mathrm{k}_{\mathrm{x}}-\mathrm{k}_{\mathrm{y}}$ is shown in Fig. 9, in which 2-D Fourier transform $\left|\mathrm{E}_{\mathrm{y}}\left(\mathrm{k}_{\mathrm{x}}, \mathrm{k}_{y}\right)\right|$ is plotted in the $\mathrm{m}-\mathrm{n}$ plane. Note that $\mathrm{k}_{\mathrm{x}}=\mathrm{m} \times 2 \pi / \mathrm{L}_{\mathrm{x}}$ and $\mathrm{k}_{\mathrm{y}}=\mathrm{n} \times 2 \pi / \mathrm{L}_{\mathrm{y}}$, where $\mathrm{m}$ and $\mathrm{n}$ are the mode numbers. The noteworthy features of Fig. 9 are the following. The panel for $\mathrm{t}=200 \omega_{\mathrm{po}}^{-1}$ reveals that power in $\mathrm{E}_{\mathrm{y}}$ is concentrated in the mode $m=n=0$, implying that a constant 

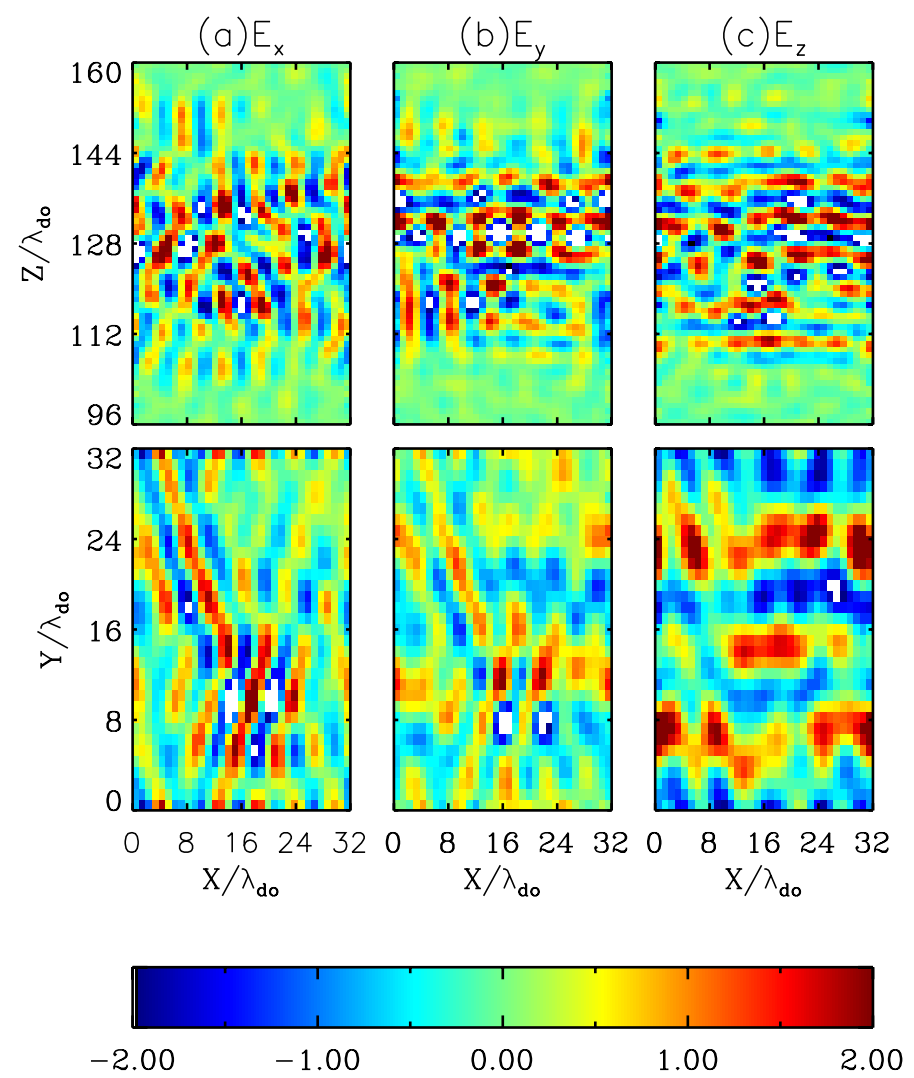

Fig. 8. Spatial structure of the fluctuations in $E_{X}$ (left column), $E_{y}$ (middle column) and $E_{z}$ (right column) are shown in the $x-z$ plan (top row) and in the $x-y$ plane (bottom row). Fluctuations are confined within the narrow current sheet as seen in the top row. In the $x-y$ plane they are superposition of a few wave modes.

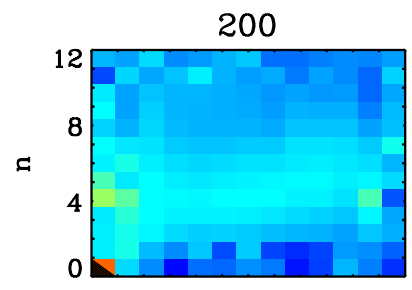$$
12
$$

$\therefore$
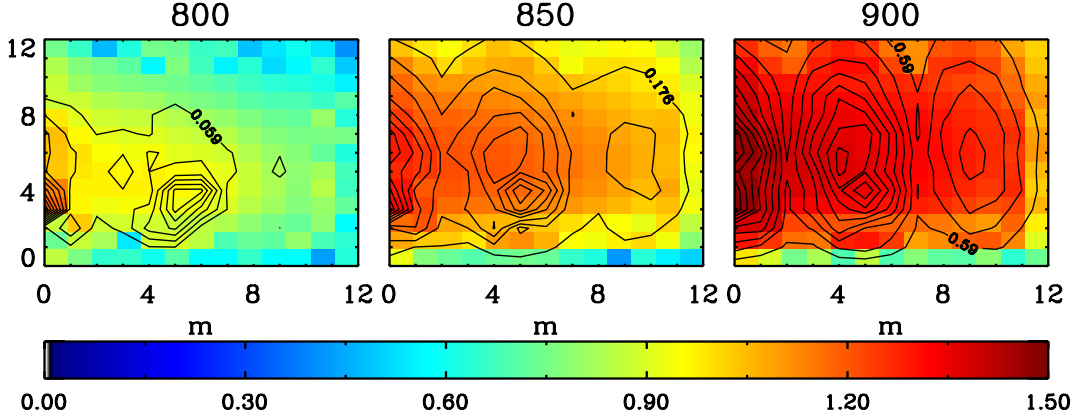

Fig. 9. Temporal evolution of the wave number spectrum of the fluctuations in $E_{y}$ in the $m-n$ plane, where $m$ and $n$ give the mode numbers according to $\mathrm{k}_{\mathrm{x}}=\mathrm{m}\left(2 \pi / \mathrm{L}_{x}\right)$ and $\mathrm{k}_{\mathrm{y}}=\mathrm{n}\left(2 \pi / \mathrm{L}_{y}\right)$. At times earlier than $\mathrm{t}=800 \omega_{\mathrm{po}}^{-1}$, the spectrum consists of narrow peaks near discrete values of $\mathrm{m}$ and $\mathrm{n}$, but at later times when reconnection sets in the spectrum broadens rapidly. 
$E_{y}$ dominates in the $x-y$ plane near $z=128 / \lambda_{\text {do }}$. This is found to be true for early times $t<200 \omega_{\mathrm{po}}^{-1}$. After this time spatially constant $\mathrm{E}_{\mathrm{y}}$ nearly vanishes and it becomes wave like. As we noted earlier, the constant value of $\mathrm{E}_{\mathrm{y}}$ at early times plays the key role in initiating the current in the CS by accelerating electrons in the $-\mathrm{y}$ direction. When the ES instability sets in at $\mathrm{t} \sim 300 \omega_{\mathrm{po}}^{-1}$, peaks begin to develop in $\left|\mathrm{E}_{\mathrm{y}}\left(\mathrm{k}_{\mathrm{x}}, \mathrm{k}_{\mathrm{y}}\right)\right|$ at non-zero values of $\mathrm{k}_{\mathrm{x}}$ and $\mathrm{k}_{\mathrm{y}}$; the peaks correspond to the dominant modes. One of the dominant modes is $(m=0, n=4)$. Another equally dominant mode is $(5,4)$, but the corresponding peak is quite broad straddling $m=4,5$ and 6 . The mode structure, consisting of the dominant modes, seems to last until $\mathrm{t} \sim 800 \omega_{\mathrm{po}}^{-1}$. The wavelengths of the dominant modes are $\lambda_{x} \sim \lambda_{y}=6-8 \lambda_{\mathrm{do}}$, giving $0.7<\mathrm{k}_{\mathrm{y}} \lambda_{\mathrm{do}} \sim \mathrm{k}_{\mathrm{x}} \lambda_{\mathrm{do}}<1$. We had already noted that the wavelength $\lambda_{z} \sim 8 \lambda_{\mathrm{do}}$ and $\mathrm{k}_{\mathrm{z}} \lambda_{\mathrm{do}} \sim 0.7$. Such wave numbers for the dominant modes are comparable with the wave numbers expected for the Buneman and ion acoustic instabilities given by $\mathrm{k}_{y} \sim \omega_{\mathrm{po}} / \mathrm{V}_{\mathrm{de}}$ when electron drift velocity $\mathrm{V}_{\mathrm{de}} \sim \mathrm{V}_{\text {teo }}$ (Fried and Gould, 1961). We note from Figs. 1a and $\mathrm{b}$ that in the central CS $\mathrm{V}_{\mathrm{de}} \sim \mathrm{J}_{\mathrm{y}} / \mathrm{n}_{\mathrm{e}} \sim \mathrm{V}_{\text {teo }}$ over $200<\mathrm{t} \omega_{\mathrm{po}}<600$ as $\mathrm{J}_{\mathrm{ymax}} \sim 2 \mathrm{~J}_{\text {th }}$ and $\mathrm{n}_{\mathrm{emax}} \sim 2 \mathrm{n}_{\mathrm{o}}$.

Figure 9 shows a sudden characteristic change in wave structure at $\mathrm{t} \sim 800 \omega_{\mathrm{po}}^{-1}$; the wave number spectrum considerably broadens in both $\mathrm{k}_{\mathrm{x}}$ and $\mathrm{k}_{\mathrm{y}}$, the fluctuations in $\mathrm{E}_{\mathrm{x}}$, $\mathrm{E}_{\mathrm{y}}$ and $\mathrm{E}_{\mathrm{z}}$ become spiky. The corresponding spatial distributions of electrons and ions in the $\mathrm{x}-\mathrm{y}$ plane near the CS center ( $\mathrm{z} \sim 128 \lambda_{\mathrm{do}}$ ) become highly bunched as shown in the top and bottom panels of Fig. 10, respectively. We interpret the bunching by trapping of the charged particles in potential well of the large-amplitude electrostatic instability. In a previously reported simulation with mass ration $\mathrm{M} / \mathrm{m}=16$ (Singh, 2004), we found the trapping, but it was not certain that in a plasma with real heavy mass this type of trapping could occur affecting both electrons and ions. Figure 10 shows that the trapping does occur with $\mathrm{M} / \mathrm{m}=1836$, but is delayed. However, the delay does not scale either with the ion-acoustic time scale as $(\mathrm{M} / \mathrm{m})^{1 / 2}$ or the Buneman instability time scale as $(\mathrm{M} / \mathrm{m})^{1 / 3}$. The deep trapping with $\mathrm{M} / \mathrm{m}=16$ occurs at $\mathrm{t} \sim 600 \omega_{\mathrm{po}}^{-1}$ while for $\mathrm{M} / \mathrm{m}=1836$ it does at $\mathrm{t} \sim 900 \omega_{\mathrm{po}}^{-1}$. This suggests that CDI in CS is affected by the non-uniformity in the sheet parameters across the $\mathrm{z}$ direction. We have suggested that shear in the drift velocity, $\mathrm{V}_{\mathrm{de}}(\mathrm{z})$, of the current-carrying electrons might enhance the growth of the CDI, especially when additional shear begins to develop near the CS center when CDI begins to reduce the current density and the drift velocity profile $\mathrm{V}_{\mathrm{de}}(\mathrm{z})$ becomes concave in the central CS (Singh, 2004). Figure 11 shows the evolution of $\mathrm{V}_{\mathrm{de}}(\mathrm{z})$ from the run with $\mathrm{M} / \mathrm{m}=1836$. Until $\mathrm{t} \sim 600 \omega_{\mathrm{po}}^{-1} \mathrm{~V}_{d e}(\mathrm{z})$ has a maximum value of $\mathrm{V}_{\text {demax }} \sim \mathrm{V}_{\text {teo }}$ near $\mathrm{z} \sim 128 \lambda_{\mathrm{do}}$. We note that this velocity is below the threshold for the Buneman instability because of the enhanced electron temperature $T_{\text {ey }}$. After $t \sim 700$ a minimum begins to develop near the center and soon the depth at the minimum reaches $\mathrm{V}_{\mathrm{de}} \sim 0$ by the time $\mathrm{t} \sim 900 \omega_{\mathrm{po}}^{-1}$. How this new shear affects
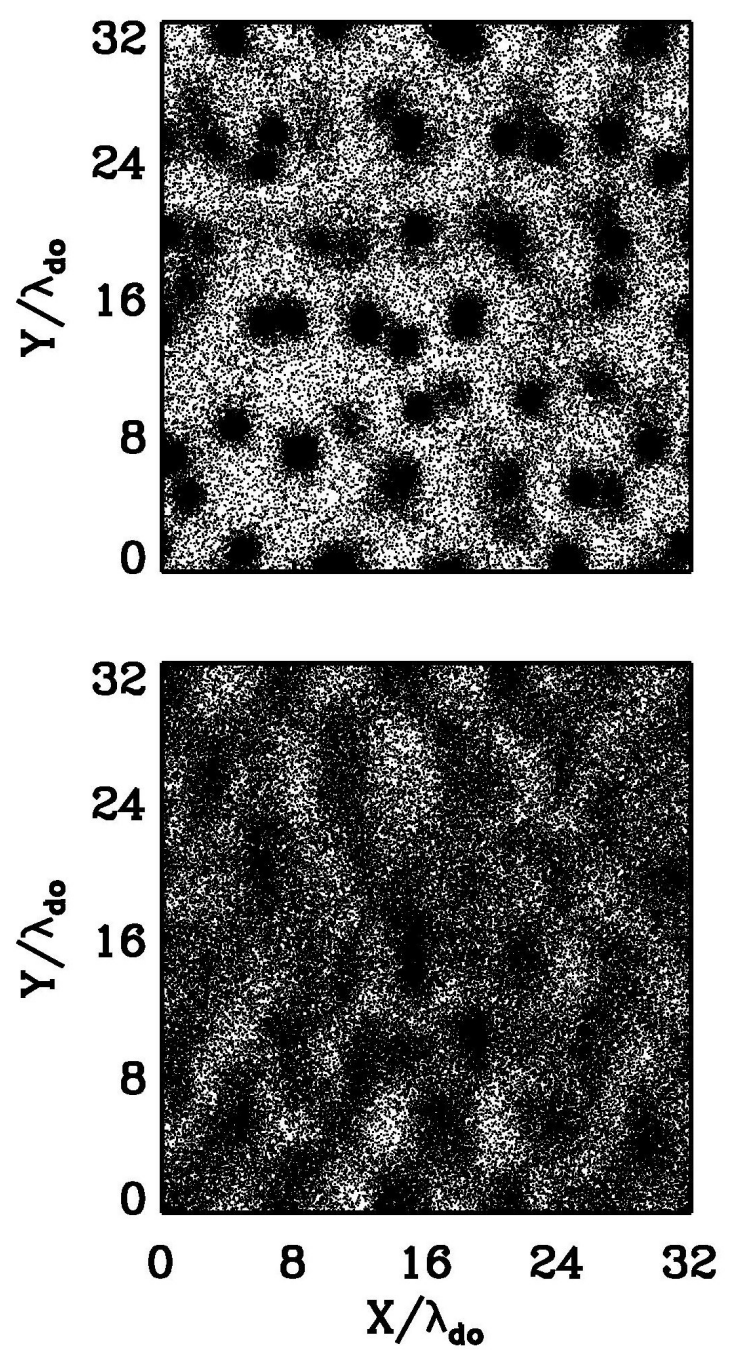

Fig. 10. Spatial distribution of electrons in the $x-y$ plane near $z=128$, showing bunching of the electrons; the bunches are the consequence of the trapping of the current-carrying electrons in the growing ES oscillations.

the CDI is not well understood. However, our simulations show that the escalation in the growth of the ES instability is accompanied by the onset of this newly sheared drift of the current carrying electrons. In addition to the developing shear in the CS center, heated electrons in the CS begin to have temperature $\mathrm{T}_{\text {ey }}$ well above the ion temperature $\mathrm{T}_{\text {iy }}$ (see Fig. 15). The resulting escalating growth of the ES instabilities and current disruption leads to magnetic reconnection. The exact mechanism causing the CDI and its evolution, including the evolution of the shear in the drift velocity of the current carriers, remains to be determined.

\subsection{Magnetic reconnection}

Figures 12a-d show the vector plots of the magnetic field in the $\mathrm{x}-\mathrm{z}$ plane. Prior to $\mathrm{t}=700 \omega_{\mathrm{po}}^{-1}$, B vectors are aligned with 


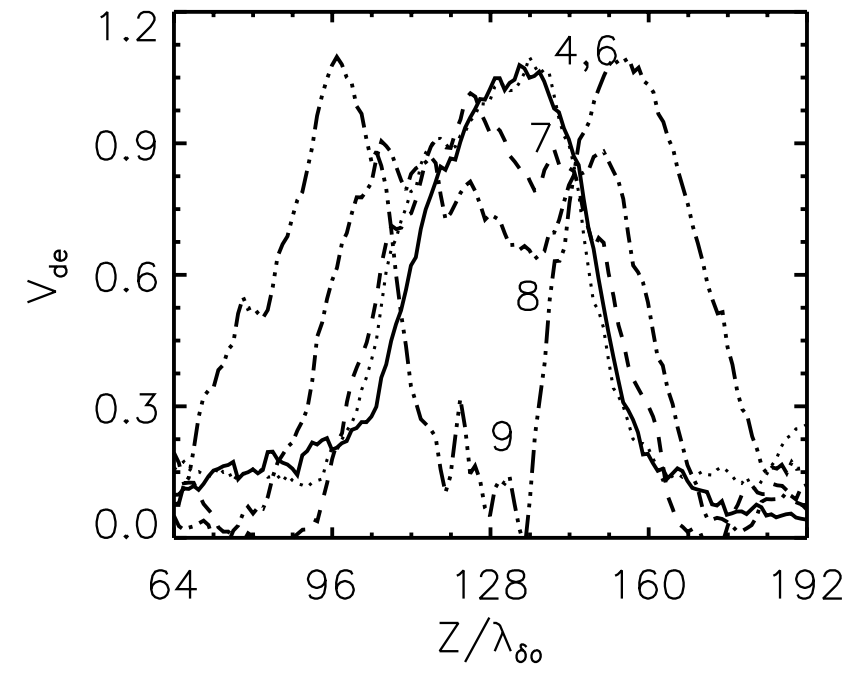

Fig. 11. Temporal evolution of the average drift of the electrons $\mathrm{V}_{\text {dey }}(\mathrm{z})$, showing that it develops a minimum in the central CS after $\mathrm{t} \sim 700 \omega_{\text {po }}^{-1}$. Before this time $\mathrm{V}_{\text {dey }}(\mathrm{z})$ has a maximum at the center.

the $\mathrm{x}$-axis all over the $\mathrm{x}-\mathrm{z}$ plane. However, we do notice that at $\mathrm{t}=700 \omega_{\mathrm{po}}^{-1}, \mathrm{~b}_{z}$ is beginning to develop along the central line $\mathrm{z}=128 \lambda_{\mathrm{do}}$, but it is quite weak. At $\mathrm{t}=800 \omega_{\mathrm{po}}^{-1}$ we see the formation of $x$ line, which is stretched over $8<x / \lambda_{\mathrm{do}_{0}}<24$. At $\mathrm{t}=850 \omega_{\text {po }}^{-1}$ the reconnected field lines are spread (Fig. 12c) over a wider range of $\mathrm{z}$ and there are formation of O-shaped structures in the reconnected region. At an even later time $\mathrm{t}=900 \omega_{\mathrm{po}}^{-1}$, the central region of reconnection continually spreads and it becomes a carpet of electromagnetic turbulence as shown in Fig. 12d. Since our simulation region is limited, we stop the simulation at $\mathrm{t}=1000 \omega_{\mathrm{po}}^{-1}$. However, we find that the magnetic reconnection begins along the neutral line and spreads outward. We examine the spatial structure of the $b_{z}$ component of the magnetic perturbation, which affects the reconnection. Figure 13a shows the evolution of $\mathrm{b}_{z}(\mathrm{x}, \mathrm{y}=16, \mathrm{z}=128)$; curves corresponds to the four times of the vector plots in Fig. 12. Note that the amplitude of the spatial oscillations in $b_{z}$ grows until $t \sim 900 \omega_{\mathrm{po}}^{-1}$. The 2DFourier spectrum of $\mathrm{b}_{z}(\mathrm{x}, \mathrm{y}, \mathrm{z}=128)$, namely, $\mathrm{b}_{z}\left(\mathrm{k}_{\mathrm{x}}, \mathrm{k}_{y}\right)$, is plotted in Figs. 13b and $\mathrm{c}$ at $\mathrm{t}=850$ and $900 \omega_{\mathrm{po}}^{-1}$. Note that $\mathrm{m}$ $\left(\mathrm{k}_{\mathrm{x}}=\mathrm{m} \times 2 \pi / \mathrm{L}_{x}\right)$ and $\mathrm{n}\left(\mathrm{k}_{\mathrm{y}}=\mathrm{n} \times 2 \pi / \mathrm{L}_{y}\right)$ are the mode numbers along the $\mathrm{x}$ and $\mathrm{y}$ directions, respectively. The spectra show that dominant mode has $\mathrm{m}=1$ and $\mathrm{n}=0$. The mode number $\mathrm{n}=0$ implies that $\mathrm{b}_{z}$ does not vary significantly with $\mathrm{y}$, the direction of the current flow. This suggests that in the midst of the three dimensional nature of the electrostatic CDI, the developing reconnection shows 2-D behavior in the $\mathrm{x}-\mathrm{z}$ plane. However, the broadness of the peak at $\mathrm{n}=0$ in the spectrum indicates that $\mathrm{b}_{z}$ might vary with $\mathrm{y}$ to some extent. The mode $\mathrm{m}=1$ implies that the electromagnetic (EM) mode facilitating the MR has one wavelength fitting in the box in the $\mathrm{x}$ direction parallel to the anti-parallel magnetic field, and it is

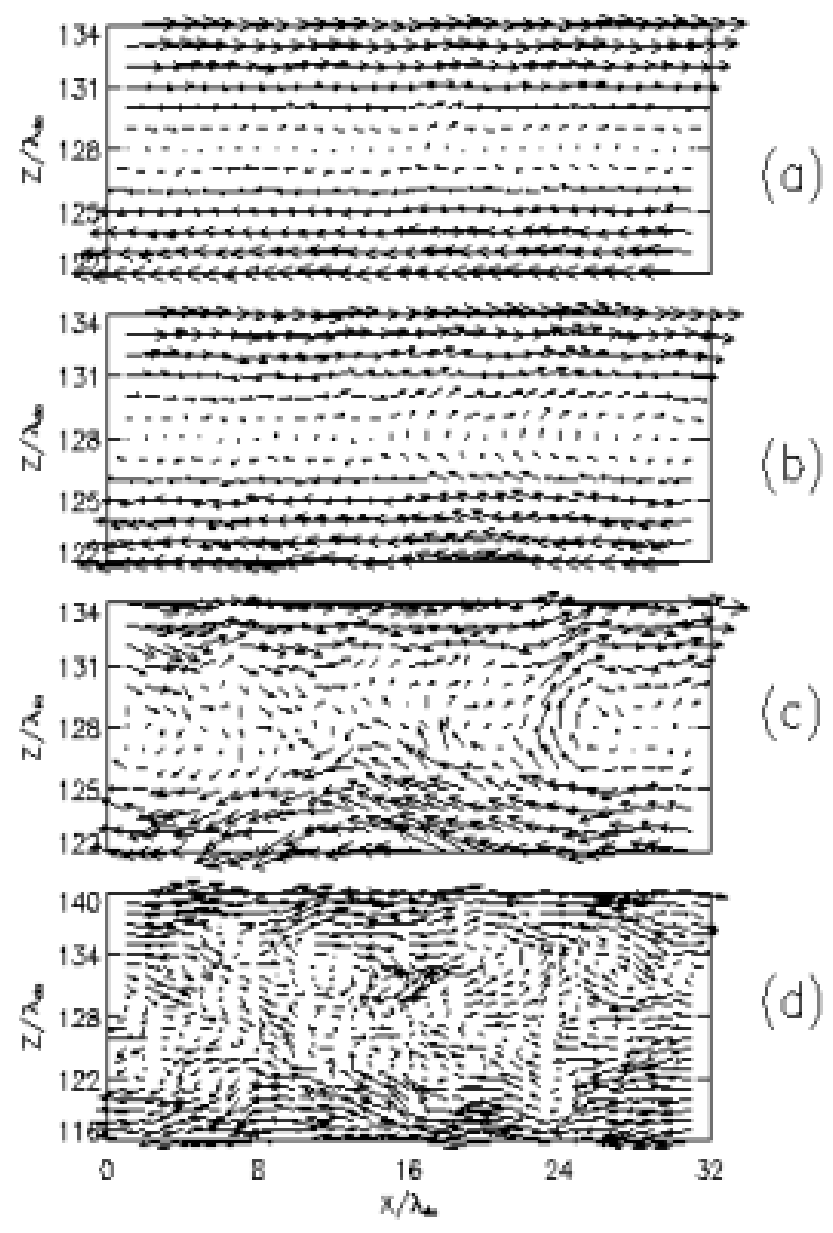

Fig. 12. Vector plots of $\mathbf{B}(x, z)$ in the $x-z$ plane at (a) $t=700 \omega_{\text {po }}^{-1}$, (b) $800 \omega_{\text {po }}^{-1}$, (c) $850 \omega_{\text {po }}^{-1}$ and $t=900 \omega_{\text {po }}^{-1}$, showing the onset of magnetic reconnection at $\mathrm{t} \sim 800 \omega_{\text {po }}^{-1}$. At times earlier than $\mathrm{t}=700 \omega_{\text {po }}^{-1}$ the $\mathbf{B}$ vectors are like the initial anti-parallel fields. At the later times when reconnection sets in, the magnetic field configuration in the central part of the current sheet undergoes a rapid change.

about $3 \lambda_{\mathrm{e}}$ long. The nature of the EM instability affecting the reconnection is examined in Sect. 3.5 on electron tearing instability (ETI).

Figure $12 \mathrm{~d}$ shows that after the onset of the magnetic reconnection, the central part of the CS is highly turbulent. We examine the effect of the turbulent magnetic fields on the CS structure and compare it with the structure at early times before the MR. Figure 14 shows a plot of $B_{x}(z)$ at $t=900 \omega_{p o}^{-1}$ along a cut at $x=16 \lambda_{\text {do }}$ in Fig. 12 d. The corresponding plots of $B_{X}(z)$ at early times can be seen in Figs. $3 a$ and $b$. The turbulent magnetic field (Fig. 12d) has transformed the smooth variation of $\mathrm{B}_{\mathrm{X}}(\mathrm{z})$ at early times into a series of steps as seen from Fig. 14. The steps appear as extremely narrow current sheets stacked together. The total width of an individual superfine current sheet is $\lambda_{\mathrm{e}}$. Such steps in the profile of the magnetic field in a reconnecting CS are clearly seen from Cluster data reported by Wygant et al. (2005). 


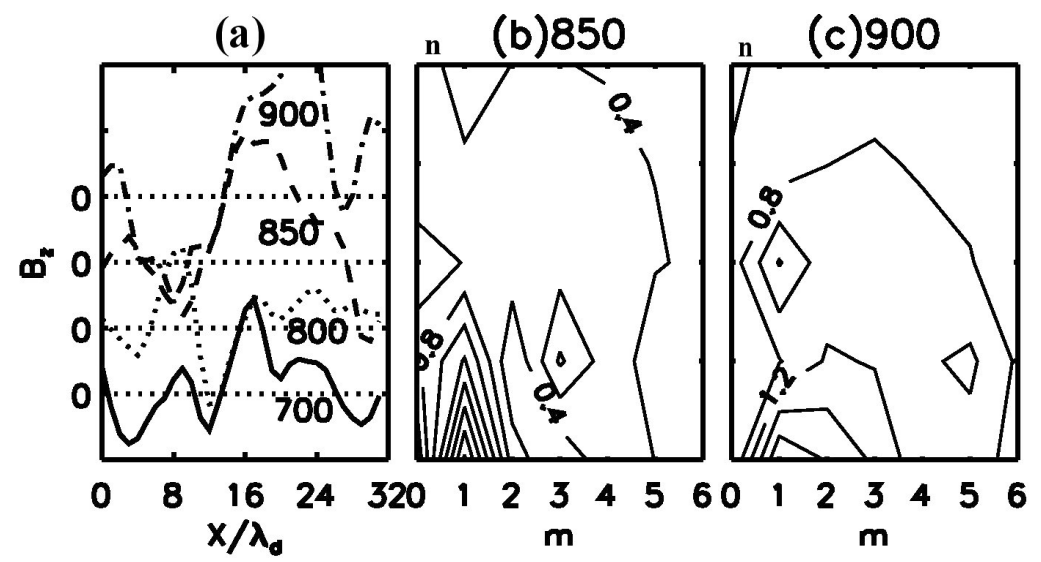

Fig. 13. (a) Temporal evolution of $b_{z}(x)$ at $z=128$ and $y=16$; the curves are for $t=700,800,850$ and $900 \omega_{\text {po }}^{-1}$ from bottom to top; note the growing amplitude of the spatial oscillation in $b_{\mathrm{x}}(\mathrm{z})$ with increasing time. The wave number spectrum $\left|\mathrm{b}_{\mathrm{z}}\left(\mathrm{k}_{\mathrm{x}}, \mathrm{k}_{\mathrm{y}}\right)\right|:(\mathbf{b}) \mathrm{t}=850 \omega_{\text {po }}^{-1}$ and $(\mathbf{c})$ $\mathrm{t}=900 \omega_{\mathrm{po}}^{-1}$.

\subsection{Electron tearing mode}

The onset of reconnection seen in Fig. 12, and the growth of the wave-like magnetic field perturbation $b_{z}(x)$ in Fig. 13a suggest that in our simulation electron tearing mode (ETM) begins to grow suddenly at $\mathrm{t} \sim 850 \omega_{\mathrm{po}}^{-1}$. The growth time of ETM is given by (Coppi et al., 1966)

$\tau \sim \pi^{-1 / 2}\left(2 \mathrm{~L} / \rho_{\mathrm{e}}\right)^{3 / 2}\left(\mathrm{~L} / \mathrm{V}_{\mathrm{te}}\right)$.

We find that at $\mathrm{t} \sim 850 \omega_{\text {po }}^{-1}$ the CS scale size $\mathrm{L}=24 \lambda_{\mathrm{do}}$. The electron Larmor radius $\rho_{e}$ greatly varies across the $\mathrm{CS}$, but we estimate that in a narrow region where reconnection begins $\left(\left|\mathrm{B}_{\mathrm{X}}\right|<0.1\right) \rho_{\mathrm{e}} \sim 15 \lambda_{\text {do. }}$. Thus we find that the growth time $\tau \sim 75 \omega_{\mathrm{po}}^{-1}$. This is approximately the time scale of the growth seen in Fig. 13a. As suggested by Coppi et al. (1966), the instability is purely growing and it occurs explosively in the simulation. The wavelength of the ETM is $\sim \mathrm{L}$, and our limited simulation size in $\mathrm{x}$ contains only one wavelength of the dominant ETM as seen from Figs. 13a-c. It is known that ETM growth rate is enhanced by the electron temperature anisotropy (Chen and Palmadesso, 1984). As we show in the next section perpendicular temperature of the electrons steadily increases over the parallel temperature until the onset of the reconnection. This might be a factor in making the growth explosive near $\mathrm{t} \sim 850 \omega_{\mathrm{po}}^{-1}$.

\subsection{Electron and ion acceleration/heating}

The panels in Fig. 15 show the evolution of $\mathrm{T}_{\mathrm{x}}, \mathrm{T}_{\mathrm{y}}$ and $\mathrm{T}_{\mathrm{z}}$, the temperatures of electrons (top) and ions (bottom) associated with their $\mathrm{x}, \mathrm{y}$ and $\mathrm{z}$ motions. The noteworthy features of Fig. 15 are the following. The ions develop a pitch angle anisotropy in their temperature with $\mathrm{T}_{\mathrm{iz}}$ being more than double of $\mathrm{T}_{\mathrm{ix}}$ and $\mathrm{T}_{\mathrm{iy}}$, which remain nearly at the initial value of $2 \mathrm{~T}_{\mathrm{o}}$ until CDI generates large-amplitude waves and begins to affect the ions for $\mathrm{t}>600 \omega_{\mathrm{po}}^{-1}$. This thermal anisotropy

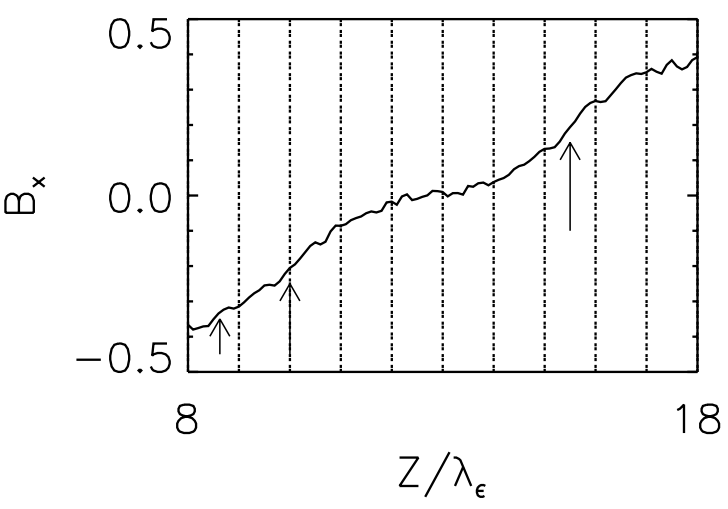

Fig. 14. $B_{x}(z)$ along a cut at $x=16 \lambda_{\text {do }}$ in Fig. $13 d\left(t=900 \omega_{\text {po }}^{-1}\right)$ is plotted to show that the EM turbulence generates step-like structures in the current sheet. The arrows show some of the steps forming superfine current sheets of total thickness $\sim \lambda_{\mathrm{e}}$.

at the early times is the consequence of ions being accelerated in the electric field $\mathrm{E}_{\mathrm{z}}$ (Fig. 4) during the time frame of the thinning of the current sheet for $\mathrm{t}<500 \omega_{\mathrm{po}}^{-1}$ (Figs. 1 and 2). Electron temperatures show a different type of thermal anisotropy with parallel temperature $T_{\mathrm{ex}}<\mathrm{T}_{\mathrm{ey}} \sim \mathrm{T}_{\mathrm{ez}}$, the perpendicular temperatures. Here parallel and perpendicular directions refer to the direction of magnetic field $B_{x}$. We see that $\mathrm{T}_{\mathrm{ex}}, \mathrm{T}_{\mathrm{ey}}$ and $\mathrm{T}_{\mathrm{ez}}$ steadily but slowly increase with time beginning at $\mathrm{t} \sim 200 \omega_{\text {po }}^{-1}$; which is nearly the time when CDI begins in the simulation. The CDI preferentially heats the electrons increasing $\mathrm{T}_{\mathrm{ey}}$, but the cyclotron motion keeps $\mathrm{T}_{\mathrm{ey}} \sim \mathrm{T}_{\mathrm{ez}}$. In contrast, since ions remain un-magnetized over the time scale of the simulation $\mathrm{T}_{\mathrm{iz}}$ remains quite different from $\mathrm{T}_{\text {iy }}$ until $\mathrm{t} \sim 800 \omega_{\text {po }}^{-1}$ when the electrostatic instability becomes explosive and reconnection sets in. After the onset of reconnection $\left(t \sim 800 \omega_{\text {po }}^{-1}\right)$, the ions in the current sheet 

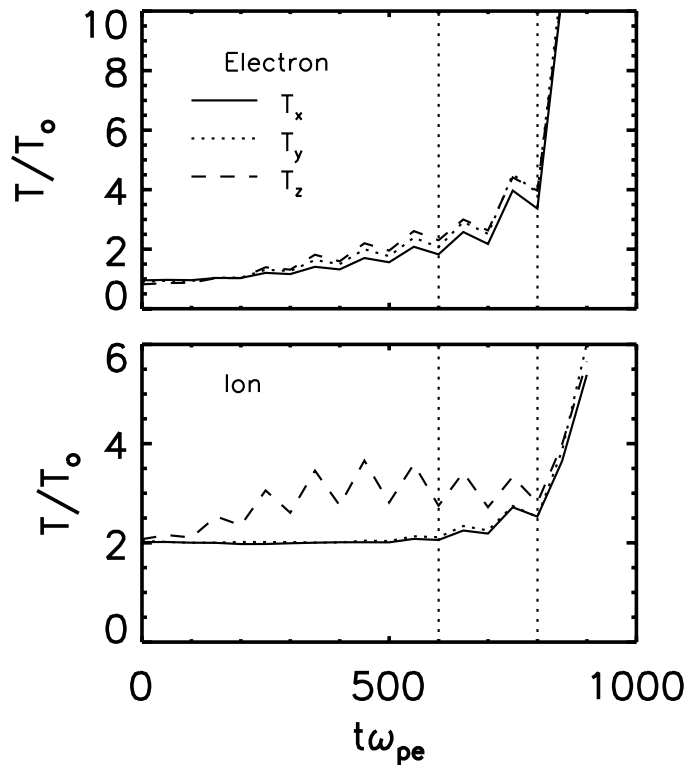

Fig. 15. Evolution of the electron (top) and ion (bottom) temperatures: The $\mathrm{x}, \mathrm{y}$ and $\mathrm{z}$ components of the temperatures are shown in the units of the normalizing temperature $\mathrm{T}_{\mathrm{O}}$.

again become almost thermally isotropic with $\mathrm{T}_{\mathrm{ix}} \sim \mathrm{T}_{\mathrm{iy}} \sim \mathrm{T}_{\mathrm{iz}}$, but with enhanced temperatures. The electron temperatures are also seen to reach isotropy with even larger temperatures after the onset of reconnection.

We find that the electron velocity distribution function $\mathrm{f}_{\mathrm{e}}\left(\mathrm{v}_{\mathrm{x}}, \mathrm{v}_{\mathrm{y}}, \mathrm{v}_{\mathrm{z}}\right)$ in the current sheet develops a complex structure after the onset of the reconnection. Figure 16 shows the evolution of the electron phase space in the $\alpha-\mathrm{V}$ plane, where pitch angle $\alpha$ is measured from the direction of $\mathbf{B}_{\mathrm{x}}$ and $V=\left(v_{x}^{2}+v_{y}^{2}+v_{z}^{2}\right)^{1 / 2}$. In Fig. 16 we have plotted $f_{e}(\alpha, V)$ on a relative scale with red and blue showing large and small $\mathrm{f}_{\mathrm{e}}$, respectively. The contours of constant $\mathrm{f}_{e}$ show that the electrons are continually heated near $\alpha=90^{\circ}$. This proceeds until $\mathrm{t} \sim 900 \omega_{\mathrm{po}}^{-1}$, after which an energetic population begins to appear at very large velocities with a broad pitch-angle distribution. Thus the $\mathrm{f}_{e}$ in the current sheet becomes dumbbell shaped and the top of the dumbbell is quickly populated with large number of electrons with relativistic speeds. Oerioset et al. (2002) have reported observations from Wind on electron acceleration in geo-tail that the most energetic electrons in the dissipation region have energies up to as high as $300 \mathrm{keV}$ and their pitch-angle distribution becomes increasingly isotropic with increasing energy. The top part of the dumbbell in Fig. 16 represents this feature of the observation from Wind.

Our primary conclusion from the discussion in this section is the following. Both electrons and ions are energized in the current sheet. A major acceleration of ions occurs during the thinning phase of the CS by the electric fields normal to the CS plane. Electrons are continually heated by CDI. However, the largest energization of both electrons and ions occur after the onset of reconnection and it occurs very rapidly in matter of few tens of electron plasma periods. Electrons in the reconnection zone achieve relativistic velocities.

\section{Conclusions and discussions}

In summary we highlight the following features of a current sheet as it forms and evolves to the onset of magnetic reconnection. Wherever possible we also highlight comparison of results from the simulation with satellite observations as well laboratory experiments.

(i) In response to an externally imposed anti-parallel magnetic field on a initially uniform and current-free plasma, a current sheet formation is triggered by the electric field $\mathrm{E}_{\mathrm{y}}$ given by Ampere's law. The electric field accelerates the electrons, which begin to carry the current.

(ii) The current carrying-electrons feel $\mathbf{J} \times \mathbf{B}$ force, which transport electrons toward the center of the current sheet. This creates space charge and electric field $E_{z}$, which converges toward the center and accelerates un-magnetized ions. $\mathrm{E}_{\mathrm{z}}$ accelerates the un-magnetized ions toward the CS center generating counter-streaming ion flow. This enhances the plasma density in the central part and reduces in the outer regions. Wygant et al. (2005) have reported that normal electric fields and ions accelerated by this field are significant features of ETCSs having scale size $\sim \lambda_{\mathrm{e}}$ as detected in the magnetotail by Cluster.

(iii) Under the influence of the converging ion flows, the current sheet thins and the thinning continues until the kinetic pressure at its center becomes as large as the sum of the magnetic and kinetic pressure near the boundaries. Initially the kinetic pressure is constant across the CS and it is $\mathrm{P}_{\mathrm{k}}=\mathrm{n}_{\mathrm{e}} \mathrm{T}_{\mathrm{ez}}+\mathrm{n}_{i} \mathrm{~T}_{\mathrm{iz}}=3 \mathrm{n}_{\mathrm{o}} \mathrm{T}_{\mathrm{o}}$ and the magnetic pressure near the boundaries is $\mathrm{P}_{\mathrm{m}}(\mathrm{z}=0)=\mathrm{P}_{\mathrm{m}}(\mathrm{z}=256)=12.5 \mathrm{~T}_{\mathrm{o}}$ and at the center $P_{m}(z=0)=0$. As the CS evolves, three factors contribute to the increase of $P_{k}$ in the central CS. They are the increase in the plasma density, increase in ion temperature $\mathrm{T}_{\mathrm{iz}}$ due to the merging of the accelerated ion streams into the central part, and the increase in $\mathrm{T}_{\mathrm{ez}}$ due to CDIs.

(iv) During the course of the thinning of the CS, magnetic field distribution $\mathrm{B}_{\mathrm{x}}(\mathrm{z})$ steepens in the central part of the CS. The resulting $\mathrm{B}_{\mathrm{x}}(\mathrm{z})$ develops three regions of distinct slopes forming three-peaked current distribution. In all three peaks current carriers are electrons. We note that these features occur at time scales $\left(<1000 \omega_{\mathrm{po}}^{-1}\right)$ much shorter than the ion cyclotron period $\tau_{c i}>23060 \omega_{\mathrm{po}}^{-1}$. The time scale of the steepening of $\mathrm{B}_{\mathrm{x}}(\mathrm{z})$ and thinning of $\mathrm{J}_{\mathrm{y}}(\mathrm{z})$ seems to occur at the speed of the fast magneto-sonic shock given by $\mathrm{V}_{\mathrm{f}}=\left(\mathrm{C}_{\mathrm{s}}^{2}+\mathrm{V}_{\mathrm{A}}^{2}\right)^{1 / 2}=0.134 \mathrm{~V}_{\text {teo. }}$. Syrovatskii et al. (1973) measured the steepening of the magnetic field distribution forming a current sheet at a time scale shorter than the ion cyclotron period in a laboratory experiment and they explained it in terms of the propagation of magneto-sonic 

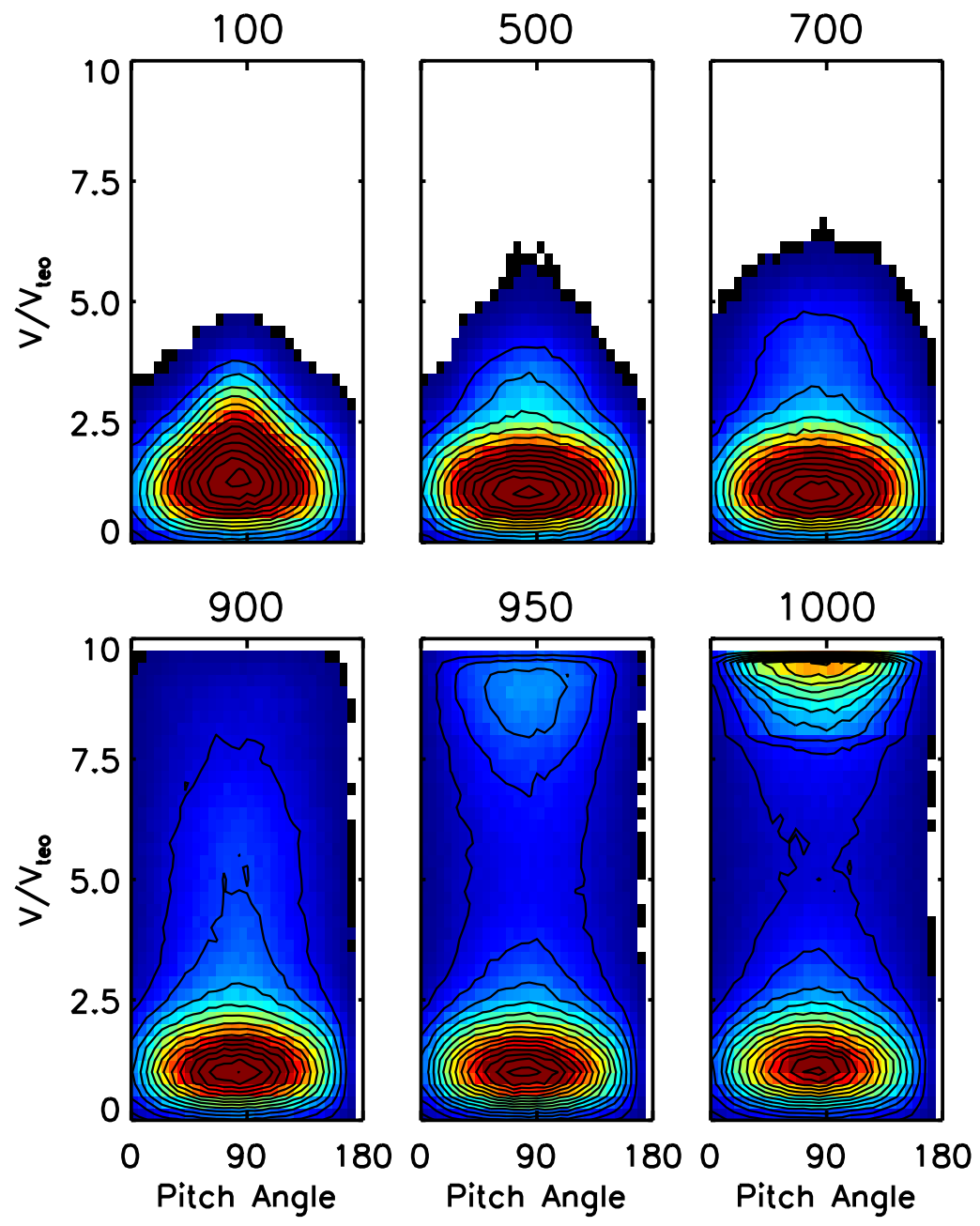

Fig. 16. Evolution of the electron velocity distribution function $\mathrm{f}_{\mathrm{e}}(\alpha, \mathrm{V})$. The times are labeled on the top of the panels. Note the formation of dumbbell shaped distribution after $\mathrm{t}=900 \omega_{\text {po }}^{-1}$ when magnetic reconnection is set-in inside the current sheet. The top part of the dumbbell is an energetic isotropic electron population reaching relativistic speeds.

waves. Wygant et al. (2005) explained the ETCS detected by Cluster in terms of standing magneto-sonic shock waves.

(v) We find that as the current sheet begins to re-broaden due to increased kinetic pressure at the CS center and the electron temperature $T_{\text {ey }}$ begins to exceed ion temperature $\mathrm{T}_{\text {iy }}$ at $\mathrm{t} \sim 700 \omega_{\mathrm{po}}^{-1}$, the growth in the ES oscillations driven by the CDI escalates. This disrupts the current in the central part of the CS by trapping the current-carrying electrons in large-amplitude oscillations. The localized current disruption causes the bifurcation of the CS. Very thin bifurcated current sheets having scale size of a few $\lambda_{e}$ were detected by Cluster (Wygant et al., 2005). We point out that the bifurcated CS seen in the 2-D simulation of Doughton et al. (2004) have structural features quite different from the Cluster data. In this 2-D simulation bifurcation is the result of current enhancement near the CS edges over the background current of the initially assumed equilibrium. On the other hand, in the Cluster data the current is nearly zero at the CS center like in our 3-D simulation (Fig. 1a); the nearly complete current disruption is caused by an explosive instability in the reconnection region.

(vi) The reconnection appears to be affected by ETI in the explosive phase of the ES instability. We find that region of the reconnected field lines spreads and becomes a carpet of electromagnetic turbulence. An interesting feature of the turbulence is that it consists of stacks of very thin current sheets of scale size $\lambda_{\mathrm{e}}$. It is interesting to point out that such stacks appear as steps in the magnetic field profiles of bifurcated currents in the Cluster data (Wygant et al., 2005).

(vii) Ion heating and acceleration occur at different stages of the evolution of the current sheet. It seems that ion acceleration by the normal electric field $\mathrm{E}_{\mathrm{z}}$ in the converging EMHD flows toward the CS center is a significant feature of a ETCS. This produces pitch-angle anisotropy in the ions' thermal structure. Besides the acceleration in the EMHD flows, ions are nearly isotropically heated in the electromagnetic 
turbulence after the onset of magnetic reconnection. The heating occurs due to large-amplitude spiky electric fields having broad wave number spectrum.

(viii) Electrons in the CS undergo a slow and steady heating by CDI until the onset of magnetic reconnection. Due to the cyclotron motion, we find that $\mathrm{T}_{\mathrm{ez}} \sim \mathrm{T}_{\mathrm{ey}}$ and these perpendicular temperatures are larger than the parallel temperature $\mathrm{T}_{\mathrm{ex}}$. This temperature anisotropy has significance for the ETI (Chen and Palmadesso, 1984). After the onset of reconnection, electrons develop a very high-energy isotropic population, in which electrons have relativistic speeds. This energetic population in conjunction with a low energy population gives the appearance of a dumbbell type of velocity distribution function in the pitch-angle-velocity phase space. Oieroset et al. (2002) have reported that electrons in the diffusion region detected by Wind became increasingly isotropic with increasing energy up to $\sim 300 \mathrm{keV}$.

In this study we have assumed that extremely thin current sheets do form in plasmas. There are observational evidence of such current sheets both from Cluster (Wygant et al., 2005; Nakamura et al., 2004). New observations of electron diffusion region reported by Mozer et al. (2005) clearly point to the existence of extremely thin current sheets. If such currents have thickness of a few times the electron skin depth, the results presented in this paper are relevant to them. For the micro-instabilities discussed here, the size of our simulation is sufficient. In simulations with longer dimensions, it is quite possible that instabilities with longer wavelengths and slower time scales might appear. Finally we point out that forthcoming NASA's Magnetospheric Multi-scale Mission, which focuses on magnetic reconnection, will provide opportunities to critically examine many of the issues discussed in this paper ranging from formation of extremely thin current sheets to plasma turbulence and magnetic reconnection in them.

Acknowledgements. This work was performed under a support from NASA grant NAG513489.

Edited by: J. Büchner

Reviewed by: G. S. Lakhina

\section{References}

Baumjohan, W. and Nakamura, R.: What is Cluster telling us about magnetotail dynamics, Proceedings of the 34th COSPAR scientific assembly, Houston, TX, USA, 10-19 Oct 2003, Proc. D3.10027-02, 1-9, 2002.

Birdsall, C. K. and Langdon, A B.: Plasma Physics via Computer Simulations, NY, McGraw- Hill, 1985.

Büchner, J. and Zelenyi, L. M.: Chaotization of electron motion as the cause of an internal magnetotail instability and substorm onset, J. Geophys. Res., 92, 13 456-13 466, 1987.

Buneman, O.: Dissipation of current in ionized media, Phys. Rev., 115, 503-517, 1959.
Cattell, C., Dombeck, J., Wygant, J., et al.: Cluster observations of electron holes in association with magnetotail reconnection and comparison to simulations, J. Geophys. Res., 110, A01211, 116, 2005.

Chen, J. and Palmadesso, P.: Tearing instability in an anisotropic neutral sheet, Phys. Fluids, 27, 1198-1206, 1984.

Coppi, B., Laval, G., and Pellat, R.: Dynamics of the Geomagnetic Tail, Phys. Rev., 16, 1207-1210, 1966.

Daughton, W., Lapenta, G., and Ricci, P.: Nonlinear Evolution of the Lower-Hybrid Drift Instability in a Current Sheet, Phys. Rev., 93, 105 004-1-105 004-4, 2004.

Drake, J. F., Swisdak, M., Cattell, C., Shay, M. A, Rogers, B. N., and Zeiler, A.: Formation of Electron Holes and Particle Energization During Magnetic Reconnection, Science, 299, 873-877, 2003.

Fried, B. D. and Gould, R. W.: Longitudinal ion oscillations in hot plasmas, Phys. Fluids, 4, 139-147, 1961.

Harris, E. G.: On a plasma sheath separating regions of oppositely directed magnetic fields, Nuovo Chimento, 23, 115-121, 1962.

Hesse, M. and Winske, D.: Electron dissipation in collisionless magnetic reconnection, J. Geophys. Res. 103, 26 479-26486, 1998.

Hesse, M., Schindler, K., Birn, J., and Kuznetsova, M.: The diffusion region in collisionless magnetic reconnection, Phys. Plasmas, 6, 1781-1795, 1999.

Hesse, M., Kuznetsova, M., and Hoshino, M.: The structure of the dissipation region for component reconnection: Particle simulations, Geophys. Res. Lett., 29(12), 1563, doi:10.1029/2001GL014714, 2002.

Horiuchi, R. and Sato, T.: Three-dimensional particle simulation of plasma instabilities and collisionless reconnection in a current sheet, Phys. Plasmas, 6, 4565-4574, 1999.

Hoshino, M., Nishida, A., Mukai, T., Saito, Y., and Yamamoto, T.: Structure of plasma sheet in magnetotail: double-peaked electric current sheet, J. Geophys. Res., 101, 24 775-24 786, 1996.

Lakhina, G. S.: On the ion tearing instability of forced current sheets, J. Gephys. Res., 98, 17 409-17 414, 1993.

Mozer, F. S., Bale, S. D., McFadden, J. P., and Torbert, R. B.: New features of electron diffusion regions observed at subpolar magnetic field reconnection sites, Geophys. Res. Lett., 32, L24102, doi:10.1029/2005GL024092, 2005.

Nakamura, R., Runov, A., Baumjohan, W., Truemann, R. A., et al.: Thin current sheets measured by Cluster at $200 \mathrm{~km}$ tetrahedron scale, http:www.glue.umd.edu/ $\sim$ sitnov/TCS/tcs_1_files/ online.htm, 2004.

Oieroset, M., Lin, R. P., Ohan, T. D., Larson, D. E., and Bale, S. D.: Evidence of Electron Acceleration up to $\sim 300 \mathrm{keV}$ in the magnetic reconnection diffusion region of Earth's magnetotail, Phys. Rev. Lett., 89, doi:10.1103/physRevLett.89.195001, 1-4, 2002.

Ozaki, M., Sato, T., Horiuchi, R., et al.: Electromagnetic instability and anomalous resistivity in a magnetic neutral sheet, Phys. Plasmas, 3, 2265-2274, 1996.

Pellat, R., Coroniti, F. V., and Pritchett, P. L.: Does ion tearing exit?, Geophys. Res. Lett., 18, 143-146, 1991.

Pritchett, P. L. and Coroniti, F. V.: Three-dimensional collisionless magnetic reconnection in the presence of a guide field, J. Geophys. Res., 109, A01220, 1-22, doi:10.1029/2003JA009999, 2004. 
Pulkkinen, T. I., Baker, D. N., Owen, C. J., et al.: Thin current sheets in the deep geomagnetotail, Geophys. Res. Lett., 20, 2427-2430, 1993.

Pulkkinen, T. I., Baker, D. N., Mitchell, D. G., et al.: Thin current sheets in magnetotail during substorms: CDAW6 revisited, J. Geophys. Res., 99, 5793-5804, 1994.

Rogers, B. N., Drake, J. F., and Shay, M. A.: The onset of turbulence in collisionless magnetic reconnection, Geophys. Res. Lett., 27, 3157-3160, 2000.

Rogers, B. N., Denton, R. E., Drake, J. F., and Shay, M. A.: Role of dispersive waves in collisionless magnetic reconnection, Phys. Rev. Lett., 87(19), doi:10.1103, 195 004-1-195 004-4, 2001.

Runov, A., Nakamura, R., Baumjohann, W., et al.: Cluster observation of bifurcated current sheets, Geophys. Res. Lett., 30, 1036, doi:10.1029/2002GL016136, 2003a.

Runov, A., Nakamura, R., Baumjohann, W., et al.: Current sheet structure near magnetic X-line observed by Cluster, Geophys. Res. Lett., 30(11), 1579, doi:10.1029/2002GL016730, $2003 \mathrm{~b}$.

Scholer, M., Sidorenko, I., Jaroschek, C. H., et al.: Onset of collisionless magnetic reconnection in thin current sheets: Threedimensional particle simulations, Phys. Plasmas, 10, 3521-3527, 2003.

Sergeev, V., Runov, A., Baumjohann, W., et al.: Current sheet flapping motion and structure observed by Cluster, Geophys. Res. Lett., 30, 1327, doi:10.1029/2002GL016500, 2003.

Silin, I. and Büchner, J.: Kinetic Instabilities of thin current sheets of thin current sheets: Results of two-and- one-half-dimensional Vlasov code simulations, Phys. Plasmas, 10, 1299-1307, 2003a.

Silin, I. and Büchner, J.: Nonlinear instability of thin current sheets in antiparallel and guided magnetic fields, Phys. Plasmas, 10, 3561-3570, 2003b.

Sitnov, M. I., Guzdar, P. N., and Swisdak, M.: A model of bifurcated current sheet, Geophys. Res. Lett., 30, 13, doi:10.1029/2003GL017218, 2003.
Shay, M. A. and Drake, J. F.: The role of electron dissipation on the rate of collisionless magnetic reconnection, Geophys. Res. Lett., 25, 3759-3762, 1998.

Shay, M. A., Drake, J. F., Swisdak, M., Dorland, W., and Rogers, B. N.: Inherently three dimensional magnetic reconnection: A mechanism for bursty bulk flows?, Geophys. Res. Lett., 30(6), 1345, doi:10.1029/2002G1016267, 2003.

Singh, N.: Instabilities in a thin current sheet, Nonlin. Processes Geophys., 11, 153-163, 2004.

Stenzel, R. L. and Gekelman, W.: Magnetic Field Line Reconnection Experiments, 1. Field Topologies, J. Geophys. Res., 86(A2), 649-658, 1981.

Syrovatskii, S. I.: Pinch sheets and reconnection in astrophysics, Ann. Rev. Astron. Astrophys., 19, 163-227, 1981.

Syrovatskii, S. I., Frank, G., and Khodhoez, A. Z.: Current distribution near the null line of magnetic field and turbulent plasma resistance, Sov. Phys. Tech. Phys., Engl. Transl., 18, 580-586, 1973.

Ugai, M.: Global dynamics and rapid collapse of an isolated current-sheet system enclosed by free boundaries, Phys. Fluids, 29(11), 3659-3667, 1986.

Wygant, T. J., Cattell, C. A., Lysak, R., et al.: Cluster Observations of an intense normal component of the electric field at a thin reconnecting current sheet in the tail and its role in the shock-like acceleration of the ion fluid into the separatix region, J. Geophys. Res., 110, A09206, 1-30, doi:10.1029/2004JA010708, 2005.

Zeiler, A., Bishkamp, D., Drake, J. F., Rogers, B. N., and Shay, M. A.: Three dimensional particle simulations of collisionless magnetic reconnection, J. Geophys. Res., 107, 1230-1239, 2002.

Zelenyi, L. M., Malova, H. V., Popov, Yu. V., et al.: Nonlinear equilibrium structure of thin current sheets: influence of electron pressure anisotropy, Nonlin. Processes Geophys., 11, 579-587, 2004. 